\title{
Predictive Encoder and Buffer Control for Statistical Multiplexing of Multimedia Contents
}

\author{
Nesrine Changuel, Student member, IEEE, Bessem Sayadi, member, IEEE, \\ and Michel Kieffer, Senior member, IEEE
}

\begin{abstract}
Statistical multiplexing of video contents aims at transmitting several variable bit rate (VBR) encoded video streams over a band-limited channel. Rate-distortion (RD) models for the encoded streams are often used to control the video encoders. Buffering at the output of encoders is one of the several techniques used to smooth out the fluctuating bit rate of compressed video due to variations in the activity of video contents. In this paper, a statistical multiplexer is proposed where a closed-loop control of both video encoders and buffers is performed jointly. First, a predictive joint video encoder controller accounting for minimum quality, fairness, and smoothness constraints is considered. Second, all buffers are controlled simultaneously to regulate the buffering delays. This delay is adjusted according to a reference delay constraint. The main idea is to update the encoding rate for each video unit according to the average level of the buffers, to maximize the quality of each program and effectively use the available channel rate. Simulation results show that the proposed scheme yields a smooth and fair video quality among programs thanks to the predictive control. A similar buffering delay for all programs and an efficient use of the available channel rate are ensured thanks to the buffer management and to the predictive closed-loop control.
\end{abstract}

Index Terms-Buffer storage, Video codecs, Predictive control, Multiplexing, Digital video broadcasting

\section{INTRODUCTION}

Video services on communication networks, such as video-on-demand, digital television, video streaming, or video conferencing, have emerged in the last few years. For example, Digital Video Broadcasting-Satellite Handheld (DVB-SH) [1] or Multimedia Broadcast Multicast Services (MBMS) [2] targets the delivery of video programs to a large audience over a broadcast channel from the service providers to mobile users.

Due to limited bandwidth resources, video programs are compressed using efficient video encoders such as MPEG 4 [3], H.264/AVC [4], or H.264/SVC [5]. The compressed programs are then multiplexed with other contents. Two encoding modes may be considered, leading to two types of multiplexing. Constant Bit Rate (CBR) encoding leads to an equal distribution of the channel rate between programs without any consideration about their respective complexity. This scheme is simple, but the quality may vary significantly with time within a single program and between programs. Encoding with Variable Bit Rate (VBR) [6] allows a simpler program to be encoded with a low rate leaving additional rate to other programs with more complex contents, e.g., action motion pictures. The purpose of statistical multiplexing (SM) [7] is then to share efficiently the channel rate between programs via a dynamic adjustment of the coding rate of each program.

Apart from the optimal use of the available channel rate, SM systems may target the satisfaction of several constraints linked to the quality-of-service (QoS) of the delivered programs, including video quality and transmission delay. For example, SM systems may be designed in such a way that

- programs are encoded with a minimum quality (minimum quality constraint [8]),

- programs are encoded with similar quality (fairness constraint [9]),

- the quality of each decoded program varies smoothly with time (smoothness constraint [10]),

- latency at the receiver side, including switching between programs, is minimized [11].

Finding a SM system able to satisfy simultaneously all these constraints in the context of video broadcasting is still a challenging task. This is mainly due to the nonstationary content of each program. Variations may be due, e.g., to scene change or to high activity within a program. In this paper, we propose a SM system that allows transmission of several video programs over a broadcast channel while taking into account the minimum quality, the fairness, and the smoothness constraints as well as the transmission delay constraint.

\section{A. Related works}

The availability of well-tuned Rate-Distortion (RD) or complexity models for each program is very useful to satisfy the previously-mentioned constraints. These models can be obtained using the feedback approach, 


\begin{tabular}{|c|c|c|c|c|c|c|c|c|}
\hline \multirow[t]{2}{*}{ SM } & \multicolumn{4}{|c|}{ Constraints } & \multicolumn{2}{|c|}{ Buffer control } & \multirow{2}{*}{$\begin{array}{l}\text { Long term } \\
\text { control }\end{array}$} & \multirow[t]{2}{*}{ Solution } \\
\hline & Channel rate & Min quality & Fairness & Smoothness & Bits & VU & & \\
\hline [11] & $\mathrm{X}$ & & & & $\mathrm{X}$ & & & Analytical \\
\hline [12] & $\mathrm{X}$ & & & & $\mathrm{X}$ & $\mathrm{X}$ & & NLO \\
\hline [13] & $\bar{X}$ & & & & $\mathrm{X}$ & & & Stochastic optimization \\
\hline$[8],[10]$ & $\mathrm{X}$ & & $(\mathrm{X})$ & $\mathrm{X}$ & $\mathrm{X}$ & & & ALO \\
\hline [14] & $\mathrm{X}$ & & $(\mathrm{X})$ & $(\mathrm{X})$ & $\mathrm{X}$ & & & Analytical \\
\hline [15] & $\bar{X}$ & & & $(\mathrm{X})$ & & & & Numerical \\
\hline [16] & $\mathrm{X}$ & & $(\mathrm{X})$ & $\mathrm{X}$ & $\mathrm{X}$ & & & Analytical \\
\hline$[17]$ & $\mathrm{X}$ & & $(\mathrm{X})$ & $(\mathrm{X})$ & $\mathrm{X}$ & & $\mathrm{X}$ & Numerical \\
\hline Here & $\mathrm{X}$ & $\mathrm{X}$ & $\mathrm{X}$ & $\mathrm{X}$ & & $\mathrm{X}$ & $\mathrm{X}$ & NLO and ALO \\
\hline
\end{tabular}

Table I

SUMMARY OF THE PROPERTIES OF VARIOUS SM SYSTEMS, NLO (LAGRANGE OPTIMIZATION WITH NUMERICAL SOLUTION), ALO (LAGRANGE OPTIMIZATION WITH ANALYTIC SOLUTION)

where RD statistics generated by the encoder are used to control the future bit-rate allocation, or using the look-ahead approach, where RD statistics computed by a preprocessor are used to adjust the bit rate prior to coding the frames in question. These two approaches are discussed in [18].

The RD trade-off of encoded streams may be adjusted by selectively discarding frames as in [19], [20] or via the encoding parameters as in [17], [21]. In the case of scalable video encoders, e.g., H.264/SVC, this control may be replaced by some packet filtering process [22], [23]. In this case, the number of transmitted enhancement layers for each frame is the control parameter. RD models, detailed in Section III, are instrumental in all cases.

Once the RD characteristics are available, one can control the encoding parameters $(\mathrm{QP}$, number of skipped frames, inter-frame prediction parameter, etc.), using a rate control algorithms. Various algorithms have been proposed in the context of single and multiple video encoding. The rate control problem for single video is addressed in [24] by comparing the quality of previously encoded frames to that of the current frame in order to interpolate the RD characteristics and to determine the appropriate encoding parameters. However, this technique accounts only for the past and may lead to violation of quality constraints. In [16], a joint encoder and statistical multiplexer of video programs is proposed. The proposed control system allows decreasing the endto-end delay and improving the average quality of compressed video by dynamically distributing the available bitrate between the video sources according to their relative complexity. A smooth video quality is achieved by allowing only small variations of the current frame quality compared to the average PSNR of previously encoded frames and by using a low-pass filter to smooth the QP variations. This method may lead, however, to difficulties in case of scene change or high motion in the video programs.

To handle the complicated inter-frame dependency problem, the RD characteristics of the frames next to the one that has to be encoded are exploited in [17]. This look-ahead approach in a SM context allows getting the $\mathrm{RD}$ characteristics of future uncoded video frames within the look-ahead window with a moderate computational complexity. Good smoothness over frames and fairness are obtained with the proposed control system, however, these two constraints are not considered explicitly in the optimization problem which makes them difficult to be achieved with other system conditions (channel rate, scene change, etc).

Among all constraints, the smoothness is the most difficult to satisfy due to the non-stationary content of video programs. This constraint has been considered in the context of single-source video coding, e.g., in [25][27], and in a SM context e.g., in [8], [10]. The SM system proposed in [10] aims at minimizing the variance of the distortion of the encoded frames. This allows getting a better video quality in average. The encoding rate is adjusted so that the rate constraint is updated according to the level of a shared buffer achieving the target smoothness distortion constraint. However, such shared buffer hinders the control of the buffering delay for each individual multiplexed program.

The buffer control is another important issue to ensure a good use of the available channel rate and to limit the video delivery delay. Buffer control has been considered, e.g., in [28]-[32]. For example, in [30] and [32], several streams are multiplexed and their transmission rates are adapted based on buffer occupancy information. This method allows a simultaneous adjustment of the rate and of the buffer occupancy, however, the buffer occupancy is controlled at a bit level which does not allow to control the transmission delay. The rate allocation method 
proposed in [12] minimizes the allocated resource while guaranteeing some QoS requirements. QoS is related to both buffer load and delay (probability of buffer overflow and delay violation). Rate control and buffering delay are also addressed in [11] where a technique for performing SM in conjunction with time slicing in DVB-H, implemented in the IP encapsulator is proposed. This method achieves a satisfactory use of the channel rate and a minimum buffering delay for all multiplexed programs. Nevertheless, in both [11] and [12], no constraint on the video quality is considered.

A summary of previous results concerning SM systems with the considered constraints is provided in Table I where $(X)$ means that the constraint is experimentally achieved with the considered scenario but not explicitly targeted.

\section{B. Main contributions}

In this paper, we introduce a SM system which performs a joint closed-loop control of video encoders and buffers. The control is performed at a video unit (VU) level to provide at any time instant a smooth quality between the VUs of a given program and bounded quality differences between the VUs of the multiplexed programs. The channel rate constraint and similar transmission delays for all programs are also targeted.

The parameters of all video encoders are adjusted for each VU using a predictive control over a window containing the previous, current, and several future VUs. This technique allows a better satisfaction of the quality constraints compared to short-term control. For that purpose, the RD characteristics of the current and future VUs have to be estimated.

The level of the buffers is adjusted via the transmission rate of each program to fully use the available channel rate and limit the buffering delay. This control is performed at the VU level contrary to most of previous works, where it is done at the bit level. Delivery and program switching delay are thus better managed. The closed-loop is obtained using a Proportional-IntegralDerivative (PID) feedback of the buffer level information to the controller of the video encoders, which allows the encoding rate constraint to be dynamically updated.

The paper is organized as follows. Section II introduces some notations and the architecture of the proposed SM system. Several state-of-art RD models are recalled in Section III before presenting the one used in the proposed system. Section IV presents the way all constraints are involved to reach good SM performance in terms of channel rate, quality, and buffering delay constraints. Section V discusses the complexity of the proposed SM system as well as implementation issues. Section VI presents the performance of the proposed system in various scenarios before concluding this work in Section VII.

Table II summarizes the notations used in this paper.

\section{ARChitecture OF THE PROPOSED SM SYSTEM}

Figure 1 presents the proposed architecture to perform SM of $N$ video programs encoded and transmitted in parallel over a broadcast channel. Programs are assumed to be transmitted over a unidirectional broadcast channel. In this context no receiver feedback is considered, and the level of the buffers of the receivers are not available.

$V_{i j}$ is the $j$-th $\mathrm{VU}$ in the $i$-th video program. All VUs are assumed to have the same duration $T$. The frame rate $F$ as well as the number of frames per VU is $N_{\mathrm{f}}$ are assumed constant with time and identical for all multiplexed programs. The $\mathrm{QP} Q_{i j}$ is the main control parameter used by each encoder to compress $V_{i j}$.

The regulation process provides one QP per VU, which is then fed to the video encoder or transcoder. When the VU is a Group of Pictures (GoP), the encoder may use the same QP for each frame in the GoP, which provides a more or less constant quality. It may also perform an adjustment of QP for each frame around the provided value to perform a $\mathrm{RD}$ optimization, using the rate control algorithm, e.g., the results in [27].

At each time instant $j$, the encoder controller determines $Q_{i j}$ so that the encoding rates $R_{i j}^{\mathrm{e}}, i=1 \ldots N$ of the $N$ encoders satisfy some dynamically updated rate constraint $R_{j}$, defined later, while satisfying the quality constraints. At each encoder output, the $i$-th buffer stores temporarily $N_{i j}^{V U}$ encoded VUs. The draining (transmission) rate $R_{i j}^{\mathrm{t}}$ from the $i$-th buffer is determined to satisfy the channel rate constraint $R_{j}^{\mathrm{c}}$ and to control the buffer delay $\tau_{i j}$ around some reference $\tau_{0}$, expressed in seconds. The differences $\tau_{i j}-\tau_{0}$ averaged over the $N$ programs denoted by $\Delta \tau_{j}$ is fed back to the encoder controller. $\Delta \tau_{j}$ is used to get the rate constraint $R_{j+1}$. Buffers and video encoders are thus controlled in a closed loop, see Figure 1.

To control the video quality, several objective and subjective video quality measurement techniques are available, see, e.g., [33]-[35] and the references therein. Here, as in most of existing works [15], [17], [22], we use the Peak-Signal-to-Noise Ratio (PSNR) of the luminance component. The PSNR of the $j$-th frame in the $i$-th program is $P_{i j}=10 \log _{10}\left(\frac{255^{2}}{D_{i j}}\right)$ where $D_{i j}$ is the average distortion of the luminance (considering a quadratic distortion measure). 


\begin{tabular}{|c|c|c|c|}
\hline Notation & Definition & Notation & Definition \\
\hline$N$ & Number of multiplexed programs & $R_{j}$ & Encoding rate constraint at time $j$ \\
\hline$N_{\mathrm{f}}$ & Number of frames per VU & $S_{j}$ & Channel state at time $j$ \\
\hline$T$ & Video unit duration & $N_{i j}^{\mathrm{v}}$ & Number of VU in buffer $i$ at time $j$ \\
\hline$i$ & Index of the video program & $B_{i j}$ & Bit level of the $i$-th buffer at time $j$ \\
\hline$j$ & Time index & $\tau_{i j}$ & Buffer delay in the $i$-th buffer at time $j$ \\
\hline$V_{i j}$ & Video unit of the $i$-th program at time $j$ & $\tau_{0}$ & Reference delay \\
\hline$F_{i}$ & Frame rate of program $i$ & $\Delta \tau_{j}$ & Average delay deviation among programs at time $j$ \\
\hline$Q_{i j}$ & Quantization parameter of the $i$-th program at time $j$ & $\Delta P_{i j}^{\mathrm{s}}$ & Smoothness constraint \\
\hline$R_{i j}^{\mathrm{e}}$ & Encoding rate of the $i$-th program at time $j$ & $\Delta P_{i j}^{\mathrm{f}}$ & Fairness constraint \\
\hline$P_{i j}$ & PSNR of the $i$-th program at time $j$ & $K_{\mathrm{p}}$ & Proportional gain in the control system \\
\hline$R_{i j}^{\mathrm{l}}$ & Transmission rate of the $i$-th program at time $j$ & $K_{\mathrm{i}}$ & Integral gain in the control system \\
\hline$R_{j}^{\mathrm{c}}$ & Channel rate at time $j$ & $K_{\mathrm{d}}$ & Derivative gain in the control system \\
\hline
\end{tabular}

Table II

NOTATIONS

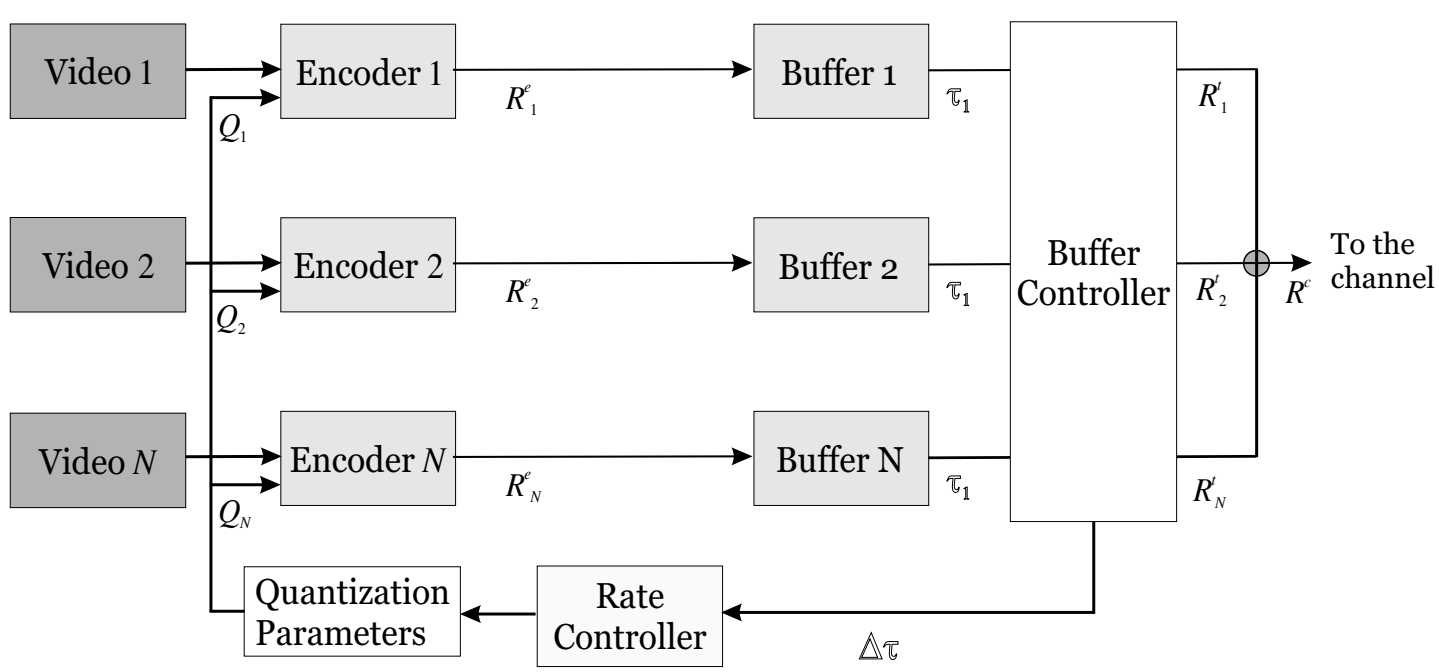

Figure 1. Proposed closed-loop statistical multiplexing system

\section{RATE AND DISTORTION MODELS}

Parametric RD models of the encoded VUs are very useful to perform an efficient rate control in the context of QoS-constrained SM. First RD models were proposed in the context of video encoding, see, e.g., [21], [36][42]. Different encoding parameters are considered such as frame rate, QP, number of skipped frames, inter-frame prediction parameters, or size of the GoP. These models may be readily used in the context of SM.

$\mathrm{RD}$ models may be grouped into independent and dependent models. In the first family, the RD characteristic of each VU is assumed independent of that of the other VUs. A simple model with few parameters is usually obtained, where the rate and distortion are the logarithm [38], the power [39], or the exponential [42] of some input parameters. Such models are quite efficient to represent the RD characteristics of a VU which consists of a whole GoP, or of INTRA-coded frames when a VU is a frame. The dependent RD models account for the impact of the RD characteristics and coding parameters of a given $\mathrm{VU}$ on the VUs taking the latter as reference, see [24], [43], [44]. Dependent $\mathrm{RD}$ models require usually more $\mathrm{RD}$ measurements to fit their parameters than independent RD models. A better accuracy is obtained at a higher computational complexity.

In what follows, a VU represents a GoP and thus, we focus on independent RD models.

\section{A. Previous results}

Most independent RD models are parametric with parameter values estimated from several encoding trials for each frame as in [36], [37] or each GoP as in [40], [41].

Linear [45] and quadratic [46] models are proposed to evaluate the rate as a function of $\rho$, the proportion of null coefficients of a quantized block in the transform domain. To be used, these models require the dependence of $\rho$ with the value of the encoding parameters, as shown in [16]. 
Inspired from [47], [48] proposed the following model for H.264/AVC at the Macroblock (MB) level

$$
\begin{aligned}
& R\left(Q_{\text {step }}\right)=\left(\frac{a_{1}}{Q_{\text {step }}}+\frac{a_{2}}{Q_{\text {step }}^{2}}+a_{3}\right)\left(a_{4} M+a_{5}\right)(1) \\
& D\left(Q_{\text {step }}\right)=a_{6} Q_{\text {step }}
\end{aligned}
$$

where $\mathbf{a}=\left(a_{1} \ldots a_{6}\right)$ is the vector of parameters, $Q_{\text {step }}$ indicates the quantization step obtained from the QP $Q$ as $Q_{\text {step }}=2^{(Q-4) / 6}$ and $S$ is the Mean Absolute Difference (MAD) of the collocated MB in the previous frame. This model requires a large number of encoding trials to be accurately tuned.

In [21] and [49], the following models have been proposed

$$
\begin{aligned}
P(Q) & =a_{\mathrm{P}} Q+b_{\mathrm{P}}, \\
R(Q) & =a_{\mathrm{R}} \exp \left(-b_{\mathrm{R}} Q\right),
\end{aligned}
$$

for the PSNR (in $\mathrm{dB}$ ) and the rate as a function of the QP $Q$. This model provides a good fit at a GoP level as illustrated in [49], and its parameters $\left(a_{\mathrm{P}}, b_{\mathrm{P}}, a_{\mathrm{R}}, b_{\mathrm{R}}\right)$ may be updated recursively to limit the number of encoding trials required to get an accurately tuned model.

Due to its moderate complexity, the model (4)-(3) has been considered in what follows.

\section{FORMULATION OF THE JOINT CONTROL AS AN OPTIMIZATION PROBLEM}

As introduced in Section II, the aim of the proposed SM system is at each time instant $j$ to provide quantization parameters $Q_{i j}$ to the video encoders and transmission rate $R_{i j}^{\mathrm{t}}$ to the buffers for all programs, while satisfying some QoS constraints. Due to variations of the RD characteristics of video contents, some shortterm decisions taken at time $j$, considering only the state of the system at time $j$, may lead to violations of the constraints at some time instant $j^{\prime}>j$.

The solution proposed for this problem is to perform at each time instant $j$ the control of the video coders taking into account the past control decisions, and the impact the current decision may have on future VUs in each program. For that purpose, a control window of $W$ VUs is considered for each program. This windows allows to take into account decisions taken for the encoding of the $j$-1-th (past) VU and decisions to be taken for the $W-2$ next VU (from $j+1$ to $j+W-2$ ), see Figure 2 . When $W=1$, only the current $\mathrm{VU}$ is involved in the optimization problem .

At time $j$ and for each program $i$, the encoder controller evaluates a vector $\mathbf{Q}_{i}^{(j)}=\left(Q_{i j}^{(j)} \ldots Q_{i j+W-2}^{(j)}\right)$. Only the parameter $Q_{i j}^{(j)}$ evaluated for VU $j$ is applied

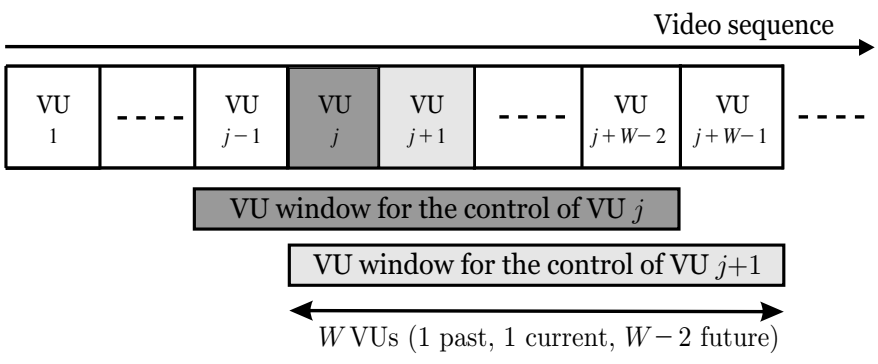

Figure 2. Predictive control involving $W$ VUs

at time $j$, the parameters $Q_{i j+k}^{(j)}$ evaluated for future VUs, $k=1 \ldots W-2$, are not applied but updated at the next time steps. This foresighted control allows choosing a value for the control parameters $\mathbf{Q}_{i}^{(j)}$ that satisfies the constraints at time $j$ and for which one knows that there exists values $Q_{i j+k}^{(j)}$ such that the constraints are also satisfied for the future time instants considered in the control window.

No predictive control is performed for the buffers following the encoders, but their level is fed back to the video encoder controller, see Figure 1.

In the following sections, the joint encoder and buffer control problem is formulated as a constrained optimization problem.

\section{A. Cost function}

The aim of the proposed SM system is to maximize the average quality of the broadcasted video programs. The considered cost function

$$
\left(\widehat{\mathbf{Q}}_{1}^{(j)} \ldots \widehat{\mathbf{Q}}_{N}^{(j)}\right)=\arg \max _{\mathbf{Q}_{1}^{(j)} \ldots \mathbf{Q}_{N}^{(j)}} \sum_{k=j}^{j+W-2} \gamma^{k-j} \sum_{i=1}^{N} P_{i k}\left(Q_{i k}^{(j)}\right)
$$

allows performing the maximization of a discounted sum of PSNRs over the control window of $W$ VUs. The discount factor $0<\gamma \leqslant 1$ provides more weight to the PSNR of current VUs for which the channel conditions and the buffer levels are well known, contrary to future PSNRs for which they are less precisely determined.

\section{B. Rate constraints}

Several rate constraints have to be satisfied, as introduced in Section II. To be stated, they require the introduction of a channel model.

1) Channel model: In the considered scenario, the bandwidth (and rate) allocated to the broadcast channel may vary with time, as in [50]. These variations may be due to concurrent services, which may leave more or less resources to the broadcast service and are readily 
considered via the Orthogonal Frequency Division Multiple Access (OFDMA) technique proposed, e.g., in the Long Term Evolution (LTE) standard [51].

The state of the broadcast channel is assumed to vary slowly with time and to be represented by a first-order Markov sequence $\left\{S_{j}\right\}$ [52], [53] with values between 1 and $n$ corresponding to $n$ channel rates $R^{1} \ldots R^{n}$ (these rates are before channel coding). $S_{j}=k$ means that the available transmission rate between time $j$ and $j+1$ is $R^{k}$. The state transition probabilities $p_{h, k}=p\left(S_{j}=h \mid S_{j-1}=k\right)$, as well as the values of the rates are assumed known a priori, they may also be estimated on-line. When performing the control at time $j$, the realization of $S_{j}$ is also assumed to be known.

2) Encoder rate constraints: In average, the sum of the encoding rates should be equal to the available transmission rate on the channel (channel rate in what follows). Thanks to the buffers, some rate variations may be tolerated. In the proposed scheme, at time $j$, a dynamically updated encoder rate constraint $R_{j}$ is provided by the buffer controller to the encoder controller, leading to

$$
(1-\varepsilon) R_{j} \leqslant \sum_{i=1}^{N} R_{i j}^{\mathrm{e}}\left(Q_{i j}^{(j)}\right) \leqslant(1+\varepsilon) R_{j}
$$

where $\varepsilon$ is a parameter that allows relaxing the encoder rate constraint. Having $\varepsilon=0$ leads to an equality constraint. When $\varepsilon>0$, the encoder rate constraint becomes an inequality, which facilitates the satisfaction of other constraints. Since it is rather difficult to accurately anticipate the future buffer levels, the encoder rate constraints for VUs between time $j+1$ and $j+W-2$ are taken as the expected value of the channel rate, knowing the current channel state $S_{j}$. Thus, the following constraints are introduced when performing the predictive control at time $j$

$$
\left\{\begin{array}{l}
(1-\varepsilon) E\left(R_{j+k}^{\mathrm{c}} \mid S_{j}\right) \leqslant \sum_{i=1}^{N} R_{i j+k}^{\mathrm{e}}\left(Q_{i j+k}^{(j)}\right) \\
\sum_{i=1}^{N} R_{i j+k}^{\mathrm{e}}\left(Q_{i j+k}^{(j)}\right) \leqslant(1+\varepsilon) E\left(R_{j+k}^{\mathrm{c}} \mid S_{j}\right)
\end{array}\right.
$$

for $k=1 \ldots W-2$, with $R_{j}^{\mathrm{c}}=R^{S_{j}}$. Satisfying (7) requires at time $j$ the availability of the RD characteristics of VUs at time $j+k, k=1 \ldots W-2$. This introduces a constant additional transmission delay $(W-2) T$ due to the buffering of $W-2$ future VUs.

3) Transmission rate and delay constraints: For each buffer, the transmission rates $R_{i j}^{\mathrm{t}}$ at time $j$ are chosen to fully use the channel rate and provide an equal buffering delay to the $N$ programs. The latter constraint leads to an average switching delay between programs (at least for what concerns the time to get a new INTRA-coded frame) independent of the target program ${ }^{1}$, and to control the delivery delay.

At time $j$, the transmission rates $R_{i j}^{\mathrm{t}}$ have thus to be such that

$$
\sum_{i=1}^{N} R_{i j}^{\mathrm{t}}=R_{j}^{\mathrm{c}}
$$

and having equal delays among programs leads to

$$
\tau_{i j+1}=\tau_{i^{\prime} j+1}, i, i^{\prime}=1 \ldots N .
$$

The delay $\tau_{i j+1}$ in buffer $i$ at time $j+1$ is difficult to determine accurately in general, since the buffers are drained bit-by-bit. Assuming that at time $j$, the bits of the encoded VU are regularly fed to the buffer with a rate $R_{i j}^{\mathrm{e}}$ and that it is regularly drained with rate $R_{i j}^{t}$, the buffer level in bits $B_{i j+1}$ at time $j+1$ is

$$
B_{i j+1}=B_{i j}+\left(R_{i j}^{\mathrm{e}}-R_{i j}^{\mathrm{t}}\right) T .
$$

One gets the following estimate of $\tau_{i j+1}$

$$
\tau_{i j+1}=\frac{B_{i j+1}}{\bar{R}_{i j}^{\mathrm{e}}}
$$

where $\bar{R}_{i j}^{\mathrm{e}}$ is an estimate of the average rate at which the VUs remaining in the buffer have been encoded. It may be evaluated, e.g., using a moving average

$$
\begin{aligned}
\bar{R}_{i 1}^{\mathrm{e}} & =R_{i 1}^{\mathrm{e}} \\
\bar{R}_{i j}^{\mathrm{e}} & =\alpha R_{i j}^{\mathrm{e}}+(1-\alpha) \bar{R}_{i j-1}^{\mathrm{e}},
\end{aligned}
$$

where $\alpha<1$ is some forgetting factor.

Combining (8), (9), (10), and (11), one obtains

$R_{i j}^{t}=R_{i j}^{\mathrm{e}}+\frac{B_{i j}}{T}+\frac{\bar{R}_{i j}^{\mathrm{e}}}{\sum_{k=1}^{N} \bar{R}_{k j}^{\mathrm{e}}}\left(R_{j}^{\mathrm{c}}-\sum_{k=1}^{N} R_{k j}^{\mathrm{e}}-\frac{1}{T} \sum_{k=1}^{N} B_{k j}\right)$.

for $i=1 \ldots N$.

With (14), one gets similar delays among programs. In practice, these delays are not strictly equal due to the approximations of the average encoding rate considered in (13). This average delay has to remain close to some reference delay $\tau_{0}$, which is chosen not too large to limit the global delivery delay, but not too small to mitigate the variations with time of the RD characteristics of video programs and of the channel rate.

At time $j$, the average delay deviation $\Delta \tau_{j}$ of each buffering delay $\tau_{i j}$ from $\tau_{0}$ is

$$
\Delta \tau_{j}=\frac{1}{N} \sum_{i=1}^{N}\left(\tau_{i j}-\tau_{0}\right) .
$$

\footnotetext{
${ }^{1}$ MBMS service requires less than 1 second delay when switching between two video programs [54].
} 
When $\Delta \tau_{j}>0$, the buffering delays are in average higher than the reference level $\tau_{0}$ and the encoding rate of the next VUs should be reduced. When $\Delta \tau_{j}<0$, the buffers are draining too fast and the encoding rate may be temporarily increased. We propose here to evaluate an updated encoding rate constraint $R_{j}$ at the buffer controller using a Proportional Integral Derivative (PID) control [55]

$R_{j}=R_{j}^{c}\left(1-K_{\mathrm{p}} \Delta \tau_{j}-K_{\mathrm{i}} \sum_{k=1}^{j} \Delta \tau_{k}-K_{\mathrm{d}}\left(\Delta \tau_{j}-\Delta \tau_{j-1}\right)\right)$,

and to feed it back to the encoder controller. In (16), $K_{p}$ is the Proportional (P) gain, $K_{i}$ the Integral (I) gain, and $K_{d}$ is the Derivative (D) gain. It is well known that $\mathrm{P}$ control cannot eliminate steady-state error. Usually, the steady-state error decreases when $K_{p}$ increases. However, a large $K_{p}$ may lead to instability. The contribution from the I term is proportional to accumulated errors, and aims at canceling the steady-state error. The D term is used to reduce the magnitude of the overshoot produced by the I term and to improve the closed-loop stability. Various methods have been proposed to tune the PID parameters, see, e.g., [56].

The control in (16), allows a regulation of the incoming flow by updating the encoding rate constraint. Such a regulation is similar to that used in the back-pressure mechanism [57]. We assume that the feedback signal $\left(\Delta \tau_{j}\right.$ or $R_{j}$ ) is available instantaneously at the encoder controller and used to select the appropriate QPs for the next VUs.

\section{Minimum PSNR constraint}

To keep an acceptable visual quality, the PSNR within a VU has to be larger than $P_{\min }$, the minimum tolerated PSNR. This leads to the constraints

$$
P_{i j+k}\left(Q_{i j+k}^{(j)}\right) \geqslant P_{\min }, i=1 \ldots N, k=0 \ldots W-2
$$

where $P_{i j+k}$ is the PSNR of the $(j+k)$-th VU in the $i$-th program. Since the future $W-2$ VUs required to formulate the constraints for $k>0$ have already been stored to satisfy the constraint presented in (7), no additional delay is introduced.

\section{Smoothness constraint}

Large PSNR variations between VUs may be visually annoying. The problem of providing video sequences with smooth quality variations has already been addressed in a single video encoding context in [25], [26], and in a SM context by [8], [10]. Here, our aim is to provide some smoothness between successive VUs, considered as GoPs. To obtain smoothness within a GoP, we refer to the works of [25], [26].

Our aim is to bound PSNR variations between successive VUs. This constraint may be relaxed in presence of scene changes, according to the results in [58]: in case of high video activity, the bitrate (and thus the quality) may be reduced to save some bitrate for parts of the video with less activity. We assume that VUs in which a scene change occurs have been detected using the methods presented, e.g., in [59], [60].

At time $j$, the absolute value of the PSNR difference between two consecutive VUs is constrained to be less than the PSNR variation bound $\Delta P_{i j}^{\mathrm{s}}$. This bound is updated when scene changes occur as follows

$$
\Delta P_{i j}^{\mathrm{s}}=\sum_{k=-\infty}^{+\infty} S_{i j-k}^{c} h_{k}^{\mathrm{s}}+\Delta P_{\min }^{s}
$$

with

$$
\begin{gathered}
h_{k}^{\mathrm{s}}=\left(\Delta P_{\max }^{\mathrm{s}}-\Delta P_{\min }^{\mathrm{s}}\right) \exp (-\lambda k) \text { if } j \geq 0 \\
h_{k}^{s}=0 \text { else }
\end{gathered}
$$

with $S_{i j}^{c}=1$ if a scene change is detected in VU at time $j$ and $S_{i j}^{c}=0$ else. $\Delta P_{\max }^{s}$ and $\Delta P_{\min }^{s}$ are respectively the maximum and the minimum PSNR variation bounds and $\lambda$ is some decay rate.

Predictive control at time $j$ takes into account the PSNR of one past (at time $j-1$ ), the current, and $W-2$ future VUs. The smoothness constraint for the $i$-th program translates into

$$
\left|P_{i j}\left(Q_{i j}^{(j)}\right)-P_{i j-1}\left(\widehat{Q}_{i j-1)}^{(j-1)}\right)\right| \leqslant \Delta P_{i j}^{\mathrm{s}},
$$

between time $j-1$, at which the control output $\widehat{Q}_{i j-1)}^{(j-1)}$ has already been applied, and time $j$. Moreover, for $k=1 \ldots W-2$, the smoothness constraint becomes

$$
\left|P_{i j+k}\left(Q_{i j+k}^{(j)}\right)-P_{i j+k-1}\left(Q_{i j+k-1}^{(j)}\right)\right| \leqslant \Delta P_{i j+k}^{\mathrm{s}},
$$

with $i=1 \ldots N$ for the future VUs.

\section{E. Inter-program fairness constraint}

Our aim is to provide multiplexed programs with quality levels of the same order of magnitude. For that purpose, the absolute value of the PSNR difference between two programs $i$ and $i^{\prime}$ is constrained to be less than some PSNR discrepancy bound $\Delta P_{\left(i, i^{\prime}\right) j}^{\mathrm{f}}$. These bounds are such that $\Delta P_{\left(i, i^{\prime}\right) j}^{\mathrm{f}}=\Delta P_{\left(i^{\prime}, i\right) j}^{\mathrm{f}}$ for all $i, i^{\prime} \in\{1 \ldots N\}$. Since at scene changes, the smoothness 
constraint is relaxed, it is necessary to update $\Delta P_{\left(i, i^{\prime}\right) j}^{\mathrm{f}}$ accordingly

$$
\Delta P_{\left(i, i^{\prime}\right) j}^{\mathrm{f}}=\sum_{k=-\infty}^{+\infty} \max \left(S_{i j-k}^{c}, S_{i^{\prime} j-k}^{c}\right) h_{k}+\Delta P_{\min }^{\mathrm{f}}
$$

with

$$
\begin{gathered}
h_{k}^{\mathrm{f}}=\left(\Delta P_{\max }^{\mathrm{f}}-\Delta P_{\min }^{\mathrm{f}}\right) \exp (-\lambda k) \text { if } j \geq 0 \\
h_{k}^{\mathrm{f}}=0 \text { else }
\end{gathered}
$$

where $\Delta P_{\max }^{\mathrm{f}}$ and $\Delta P_{\min }^{\mathrm{f}}$ are respectively the maximum and the minimum PSNR discrepancy bounds. Then, the fairness constraint at time $j$ translates into $N(N-1) / 2$ inequality constraints

$$
\left|P_{i j+k}\left(Q_{i j+k}^{(j)}\right)-P_{i^{\prime} j+k}\left(Q_{i^{\prime} j+k}^{(j)}\right)\right| \leqslant \Delta P_{\left(i, i^{\prime}\right) j+k}^{\mathrm{f}},
$$

with $k=0 \ldots W-2$ and $i, i^{\prime} \in\{1 \ldots N\}$.

\section{F. Summarized constrained optimization problem}

Considering the cost function (5) and the constraints related to the rate (6) and (7), the minimum PSNR (17), the smoothness (20) and (21), and the fairness (24), one gets the following constrained optimization problem to solve at time $j$

$$
\left(\widehat{\mathbf{Q}}_{1}^{(j)} \ldots \widehat{\mathbf{Q}}_{N}^{(j)}\right)=\arg \max _{\mathbf{Q}_{1}^{(j)} \ldots \mathbf{Q}_{N}^{(j)}} \sum_{k=j}^{j+W-2} \gamma^{k-j} \sum_{i=1}^{N} P_{i k}\left(Q_{i k}^{(j)}\right)
$$

subject to

$$
\left\{\begin{array}{l}
(1-\varepsilon) R_{j} \leqslant \sum_{i=1}^{N} R_{i j}^{\mathrm{e}}\left(Q_{i j}^{(j)}\right) \leqslant(1+\varepsilon) R_{j} \\
(1-\varepsilon) E\left(R_{j}^{\mathrm{c}} \mid S_{j}\right) \leqslant \sum_{i=1}^{N} R_{i j+k}^{\mathrm{e}}\left(Q_{i j+k}^{(j)}\right) \\
\sum_{i=1}^{N} R_{i j+k}^{\mathrm{e}}\left(Q_{i j+k}^{(j)}\right) \leqslant(1+\varepsilon) E\left(R_{j}^{\mathrm{c}} \mid S_{j}\right) \\
P_{i j+h}\left(Q_{i j+h}^{(j)}\right) \geqslant P_{\min } \\
\left|P_{i j+h}\left(Q_{i j+h}^{(j)}\right)-P_{i^{\prime} j+h}\left(Q_{i^{\prime} j+h}^{(j)}\right)\right| \leqslant \Delta P_{\left(i, i^{\prime}\right) j+h}^{\mathrm{f}} \\
\left|P_{i j}\left(Q_{i j}^{(j)}\right)-P_{i j-1}\left(\widehat{Q}_{i j-1)}^{(j-1)}\right)\right| \leqslant \Delta P_{i j}^{\mathrm{s}}, \\
\left|P_{i j+k}\left(Q_{i j+k}^{(j)}\right)-P_{i j+k-1}\left(Q_{i j+k-1}^{(j)}\right)\right| \leqslant \Delta P_{i j+k}^{\mathrm{s}} \\
\text { with } h=0 \ldots W-2, k=1 \ldots W-2 \\
\text { and }\left(i, i^{\prime}\right) \in\{1 \ldots N\}
\end{array}\right.
$$

where $\widehat{\mathbf{Q}}_{j-1}^{j-1}$ contains the QP obtained from step $j-1$ for $\mathrm{VU} j-1$.

The control of the transmission rate and buffering delay is described in Section (IV-B3).

\section{COMPLEXITY AND IMPLEMENTATION ISSUES}

\section{A. Complexity}

To analyze the complexity of the proposed SM algorithm, several aspects have to be considered.
First, at each time instant $j, \mathrm{RD}$ models have to be obtained for each VU to encode (or transcode) and for the $W-2$ VUs in the control window. In fact, only the models for the VUs at time $j+W-2$ have to be evaluated or updated from the models previously available at time $j-1$ (going up to the VUs at time $j+W-3$ ). Assuming that high-quality encoded streams are provided to the SM system, approximate RD characteristics may easily be obtained using transcoders such as that described in [61].

Then, the constrained optimization step is probably the most complex operation. The complexity depends on the number of multiplexed programs $N$ and of the size of the control windows $W$. The cost function, as well as inequality constraints are linear in the QPs. Only the rate constraint (6) is nonlinear, which make the problem much more complicated to solve. In the experimental part, see Section VI, Matlab's fmincon has been used, leading for $N=8$ programs to an average optimization time of $0.16 \mathrm{~s}$ for $W=2$ and of $0.8 \mathrm{~s}$ for $W=4$, on an Intel Core 2 Duo at $2.26 \mathrm{Ghz}$ with $1.94 \mathrm{~Gb}$ RAM. Using a linear approximation of (6) would allow the use of linear programming tools, which would drastically reduce the computational complexity of this part.

Finally, the evaluation of the transmission rates as well as the total encoding rate constraint are provided by close-form expressions. Their complexity is negligible compared to the numerical constrained optimization.

The latency introduced is equivalent to the duration of $W-1$ VUs. Thus requires to buffer $W-1$ uncoded VUs in case of encoding within the SM or high-quality encoded VUs, when considering transcoding.

Alternatively, scalable video coders (SVC) [5] may be considered. With a SVC, the RD characteristic for some rate values is easily obtained (they may be stored in packet headers). The price to be paid is an increased difficulty to satisfy accurately the fairness constraints between streams. Moreover, buffering all quality layers for $W-1$ encoded VUs is still necessary.

\section{B. Application context}

A typical application scenario for SM is the Mobile TV service delivery over evolved MBMS standard [2]. Here, we briefly describe the functional architecture of the multiplexing functions. Detailed implementation issues are not addressed.

MBMS is a point-to-multipoint interface specification for 3GPP cellular networks, which is designed to provide efficient delivery of broadcast and multicast services. For broadcast transmission, a single frequency network configuration is introduced in 3GPP LTE (Long Term Evolution) specifications which enables a time synchro- 
nization between a set of eNBs (base stations) using the same resource block.

The MBMS architecture is composed of three main entities: BM-SC, MBMS-GW and MCE. The Multicast/Broadcast Service Center (BM-SC) is a node that serves as an entry point for the content providers delivering the video sources, used for service announcements, session management.

The MBMS-GW is an entity responsible for distributing the traffic across the different eNBs belonging to the same broadcast area. It ensures that the same content is sent from all the eNBs by using IP Multicast.

The Multi-cell/multicast Coordination Entity (MCE) is a logical entity, responsible for allocation of time and frequency resources for multi-cell MBMS transmission. As in [15], we assume that the MBMS-GW periodically notifies the MCE about the resource requirements of video streams so that the resources at eNBs can be re-allocated accordingly. Therefore, the BM-SC should ensure that the encoding rate of the multiplex does not violate the already allocated resources. This is obtained thanks to the proposed SM scheme.

\section{EXPERIMENTAL RESULTS}

In this section, we evaluate the performance of the proposed joint encoder and buffer controller involving the solution of the constrained optimization problem (25) and (26).

Several video sequences are encoded with the H.264/AVC encoder in baseline profile and at the same frame rate $F=30$ frames/s. The first frame in the GoP is an I frame and the remaining frames are $\mathrm{P}$ frames. Other encoding profiles as well as other GoP structures may be considered without changing in the optimization process. However, as mentioned in Section V, estimating the parameters of the RD model for each VU of each video based on encoding trials is more time consuming with enhanced profiles or considering B frames than with the baseline profile with I and P frames only.

In Scenario 1, $N=4$ video programs are multiplexed and transmitted. Each program displays a succession of video sequences among: Soccer (V1), Container (V2), Coastguard (V3), and Hall (V4), taken from the commonly used video test sequences in CIF format. Scene changes correspond to a change of the video transmitted by a given program, see Figure 3. For example, Program 1 displays Hall (V4) till GoP 30, then switches to Container (V2), before switching to Soccer (V1) at GoP 200. GoPs of $N_{\mathrm{f}}=15$ frames are considered, thus the VU (GoP) duration is $T=0.5 \mathrm{~s}$.

In Scenario 2, $N=8$ video sequences extracted from real soccer game, film trailer, interview, video

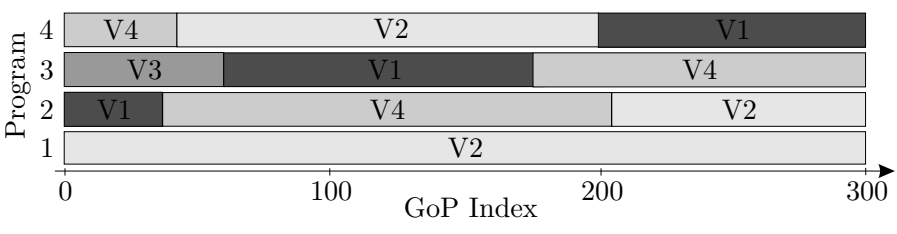

Figure 3. Videos transmitted over the four multiplexed programs

clips, game shows, weather forecasts, and cartoons are multiplexed and transmitted. GoPs of $N_{\mathrm{f}}=30$ frames are considered in this case, thus the $\mathrm{VU}(\mathrm{GoP})$ duration is $T=1 \mathrm{~s}$.

In both scenarios, each encoder receives some QP from the rate controller. The evaluation of QP for each frame of the GoP around the provided value, to get, e.g., smooth quality variations, is assumed to be managed by the RD controller at frame level implemented in the video coder.

\section{A. Scenario 1: Statistical multiplexing using four stan- dard video sequences in CIF format}

In the first set of experiments, we focus on the video encoder control process described in (25) and (26) in the context of Scenario 1.

The predictive control is performed using a control window of $W=4 \mathrm{GoPs}$, including the previous, current, and two future GoPs, and is compared to a reference scenario without predictive control $(W=2)$, but for which the smoothness constraint is still imposed between the previous and the current GoPs. The RD models, presented in Section III, are evaluated in advance for $W-1$ GoPs with two encoding trials for each GoP. Using predictive control, an encoding delay $(W-1) T$ is introduced. When $W=4$, this delay is $1.5 \mathrm{~s}$ in this case.

Scene changes are assumed known in advance for each program. The minimum tolerated PSNR is $P_{\min }=$ $30 \mathrm{~dB}$. The PSNR variations and discrepancy bounds are $\Delta P_{\max }^{s}=2.5 \mathrm{~dB}, \Delta P_{\min }^{s}=1 \mathrm{~dB}, \Delta P_{\text {max }}^{\mathrm{f}}=5 \mathrm{~dB}$, and $\Delta P_{\text {min }}^{\mathrm{f}}=2 \mathrm{~dB}$. The damping ratio is $\lambda=1.25$, leading to a negligible relaxation of the PSNR bounds after 2 to 3 VUs. In this set of experiments, $\varepsilon=0$, leading to an the encoder rate equality constraint. The size of the buffers is taken large enough to support the large bit level variations, occurring, e.g., during scene changes. Here, their size in bits is $B_{\max }=4$ Mbits. The reference delay is taken as $\tau_{0}=1 \mathrm{~s}$. Two cases are considered: (i) constant channel rate as in [11] and (ii) time-varying channel rate as in [15].

In what follows, the solutions of (25) and (26) involved in the control process are obtained numerically using Matlab's fmincon. 
1) Constant channel rate: The available channel rate is taken as $R_{j}^{\mathrm{c}}=1 \mathrm{Mbit} / \mathrm{s}$ for all time $j$. The parameters of the PID controller for the feedback from the buffer controller to the encoder controller are set to $\left(K_{\mathrm{p}}, K_{\mathrm{i}}, K_{\mathrm{d}}\right)=(0.2,0.01,0.01)$ for $W=2$ and $\left(K_{\mathrm{p}}, K_{\mathrm{i}}, K_{\mathrm{d}}\right)=(0.2,0.01,0.05)$ for $W=4$, see Section VI-A1b for more details on the tuning of the PID controller.

a) Rate and quality control: Figure 4 shows the encoding rates $R_{i j}^{\mathrm{e}}$ for each program, the total encoding rate $R_{j}^{\mathrm{e}}=\sum_{i=1}^{4} R_{i j}^{\mathrm{e}}$ and the total transmission rate $R_{j}^{\mathrm{t}}=\sum_{i=1}^{4} R_{i j}^{\mathrm{t}}$ for $W=2$ (a) and $W=4$ (b). In both cases, the transmission rates for each program is appropriately chosen so that $R_{j}^{\mathrm{t}}$ is equal to the channel rate $R_{j}^{\mathrm{c}}$. However, one sees that the jitter of the encoding rate is higher when $W=2$ than with $W=4$. Using $W=4$ allows an improved management of the complexity variations of the contents of video programs. This has also an impact on the variations of the buffer level, see Section VI-A1b.

The PSNR $P_{i j}$ of each program is represented in Figure 5 for $W=2$ (a) and $W=4$ (b). One sees that the minimum quality constraint is satisfied in both cases. One also notices that again, the PSNR jitter is higher when $W=2$ than with $W=4$, especially between GoP 200 and GoP 300. This is closely connected to the rate jitter observed in Figure 4.

To evaluate whether the smoothness constraint is satisfied, the differences between successive PSNRs $\left(P_{i j}-P_{i j-1}\right)$ for each program are represented in Figure 6 in three cases: (i) without imposing any smoothness constraint with $W=1$, (ii) with $W=2$, leading to a smoothness constraint involving only the past VU, and (iii) with $W=4$, leading to a smoothness constraint involving both past and future VUs. The smoothness constraint is less frequently violated when $W=2$ (2.75\% of time) or when $W=4(1.8 \%$ of time) than without smoothness constraint (this case is denoted $W=1$ ) (4\% of time). When $W=4$, most of the violations are due to the discrepancy between the estimated RD characteristics used in the control process and the actual RD characteristics of each video sequence. As expected, taking into account previous (and future) VUs improves the smoothness of the video sequence.

Introducing the smoothness constraint reduces the amplitude of PSNR variations, see also Figure 7, where the standard deviation of the PSNR is represented for several values of the channel rate. In the three considered cases, the standard deviation decreases with the increase of the channel rate. Taking $W>4$ with the same values of $\Delta P_{i j}^{\mathrm{f}}$ and $\Delta P_{i j}^{\mathrm{s}}$ does not provide any additional benefit in terms of variations of the PSNR.

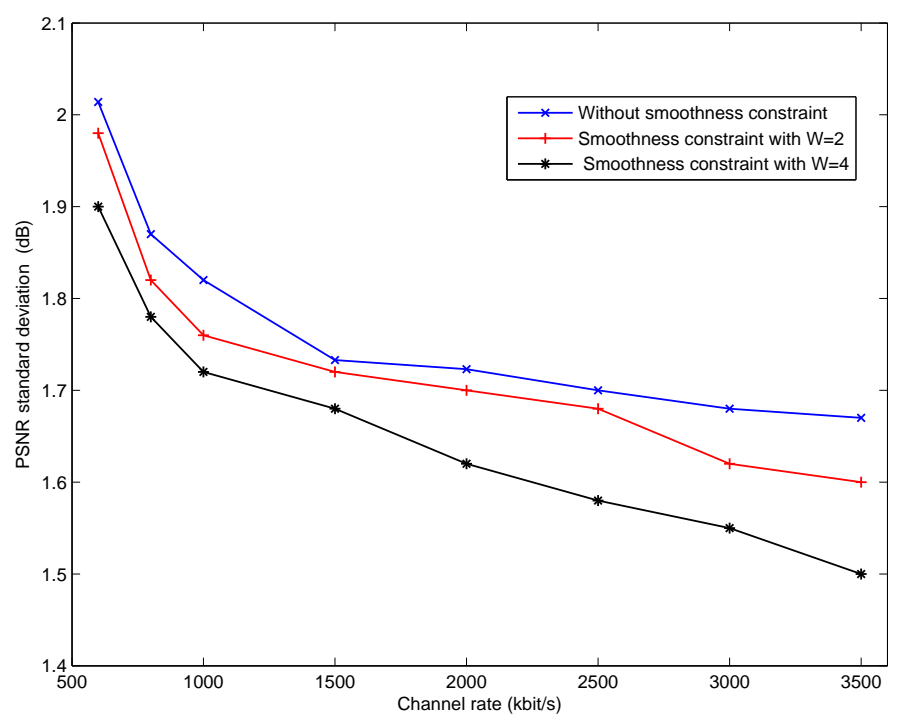

Figure 7. Standard deviation of the PSNR without imposing any smoothness constraint $W=1$, with smoothness constraint when $W=2$ and $W=4$ for different channel rates

b) Performance of the buffering delay control: This section illustrates the performance of the buffer management. The transmission rates $R_{i j}^{\mathrm{t}}$ are obtained analytically as shown in Section (IV-B3). The actual buffering delay $\tau_{i j}$ for each buffer is represented in Figure 8 for $W=2$ (a) and $W=4$ (b), using the P, PI, and PID controllers. The parameters $K_{\mathrm{p}}, K_{\mathrm{i}}$, and $K_{\mathrm{d}}$ have been tuned to minimize $e=(\Delta \tau)^{2}+\sigma_{\tau}^{2}$ where $\Delta \tau$ is the average delay discrepancy and $\sigma_{\tau}^{2}$ is the average delay variance considering the actual programs, see Section VI-A1c. The closed-loop control allows the buffering delays to be of the same order of magnitude for the four multiplexed programs, even when they display different videos with their own contents and characteristics. Although the constrained problem in (8) does not involve the reference delay $\tau_{0}$, thanks to the PID encoding rate control, the buffering delay in the four considered buffers remains around $\tau_{0}$. Moreover, independently of the choice of the controller for the encoding rate, Figure 8 shows that the delay jitter is smaller for $W=4$ than for $W=2$. This is again a consequence of the smaller rate jitter for $W=4$ than for $W=2$. The foresighted encoding rate adaptation obtained with $W=4$ to better satisfy the quality constraints has thus a direct impact on the jitter of the buffering delay.

This result is confirmed by Table III. One sees that PI encoding rate controller reduces $\Delta \tau$ compared to the $\mathrm{P}$ controller. The $\mathrm{D}$ term reduces slightly $\sigma_{\tau}^{2}$.

The forgetting factor in (13) has been set to $\alpha=0.7$, 


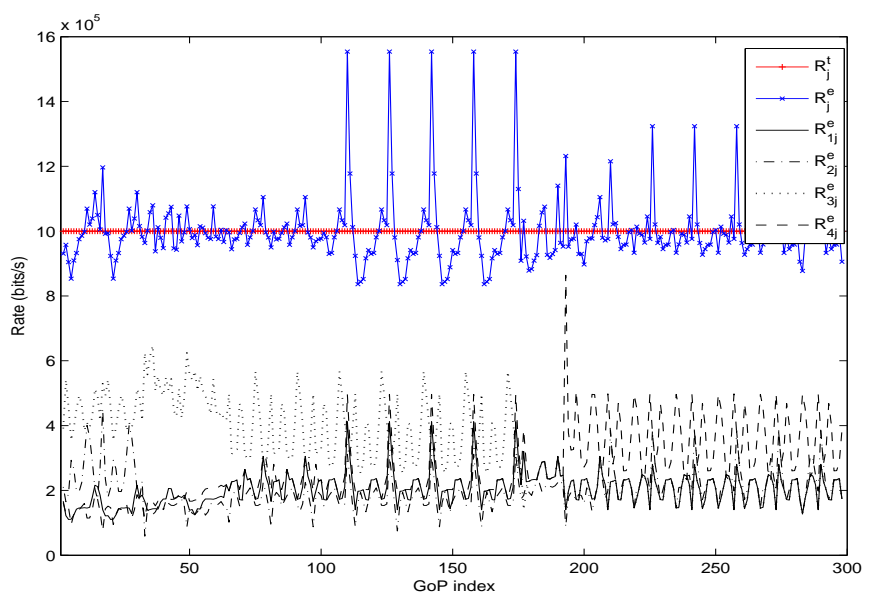

(a)

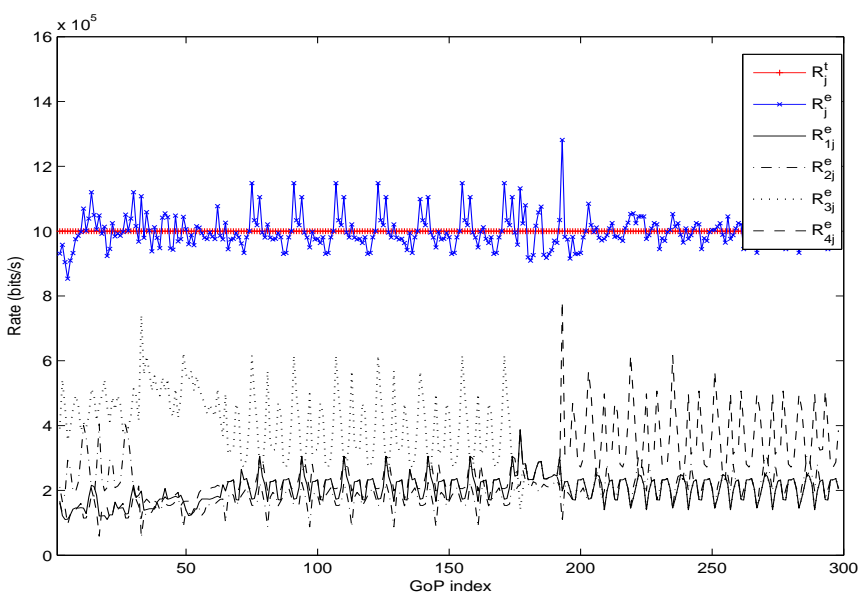

(b)

Figure 4. Evolution of the total transmission rates as well as individual and total encoding rates for the four multiplexed programs when $W=2$ (a) and $W=4$ (b)

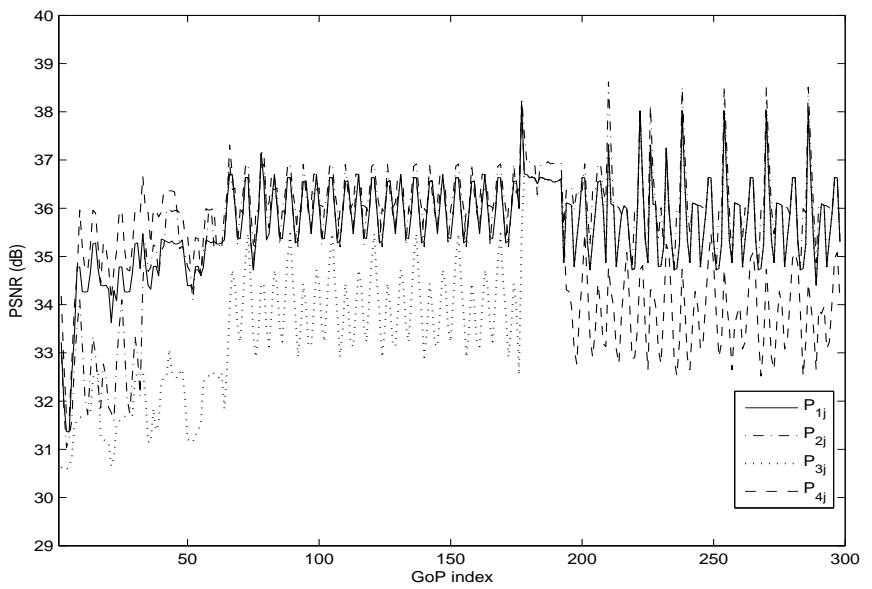

(a)

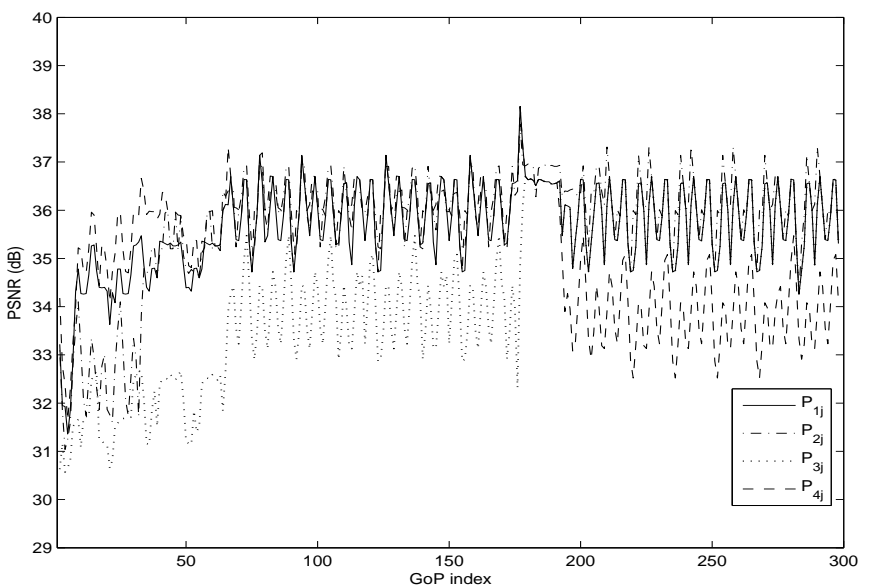

(b)

Figure 5. Evolution of the PSNR of the four multiplexed programs when $W=2$ (a) and $W=4$ (b)

\begin{tabular}{|c|c|c|c|c|c|c|c|}
\hline$K_{\mathrm{p}}, K_{\mathrm{i}}, K_{\mathrm{d}}$ & \multicolumn{3}{|c|}{$W=2$} & $K_{\mathrm{p}}, K_{\mathrm{i}}, K_{\mathrm{d}}$ & \multicolumn{3}{c|}{$W=4$} \\
\hline & $\Delta \tau$ & $\sigma_{\tau}^{2}$ & $e$ & & $\Delta \tau$ & $\sigma_{\tau}^{2}$ & $e$ \\
\hline $0.2,0,0$ & 0.02 & 0.05 & 0.05 & $0.2,0,0$ & 0.01 & 0.013 & 0.014 \\
\hline $0.2,0.01,0$ & 0.01 & 0.04 & 0.04 & $0.2,0.01,0$ & 0.003 & 0.016 & 0.016 \\
\hline $0.2,0.01,0.01$ & 0.01 & 0.04 & 0.04 & $0.2,0.01,0.05$ & 0.003 & 0.015 & 0.015 \\
\hline
\end{tabular}

Table III

SYSTEM PERFORMANCE IN TERMS OF $\Delta \tau$ AND $\sigma_{\tau}^{2}$ WHEN USING P, PI, AND PID CONTROLLERS FOR $W=2$ AND $W=4$ USING CONSTANT CHANNEL RATE

which corresponds to the smallest average relative discrepancy (less than $2 \%$ ) between the estimated delay using (11) and the actual buffer delay represented in Figure 8 .

c) Tuning of the P, PI, and PID controllers: Several techniques are available to tune PID controllers, see, e.g., [55]. Here, the parameters of the PID controller have been tuned manually using first a simplified scenario where the 4 multiplexed programs display only the first
GoP of each video in a loop. Moreover, buffers have been assumed to be initially empty. This helps to get some steady-state after a transient behavior. The evolution of the buffer delays for different values of the PID parameters and $W=4$ is presented in Figure 9. $K_{\mathrm{p}}$ is tuned first to maximize the rise speed to some equilibrium while having no overshoot. In Figure 9(a), the choice $K_{\mathrm{p}}=0.3$ appears to be a good compromise. $K_{\mathrm{i}}$ is adjusted to eliminate the offset and to minimize 

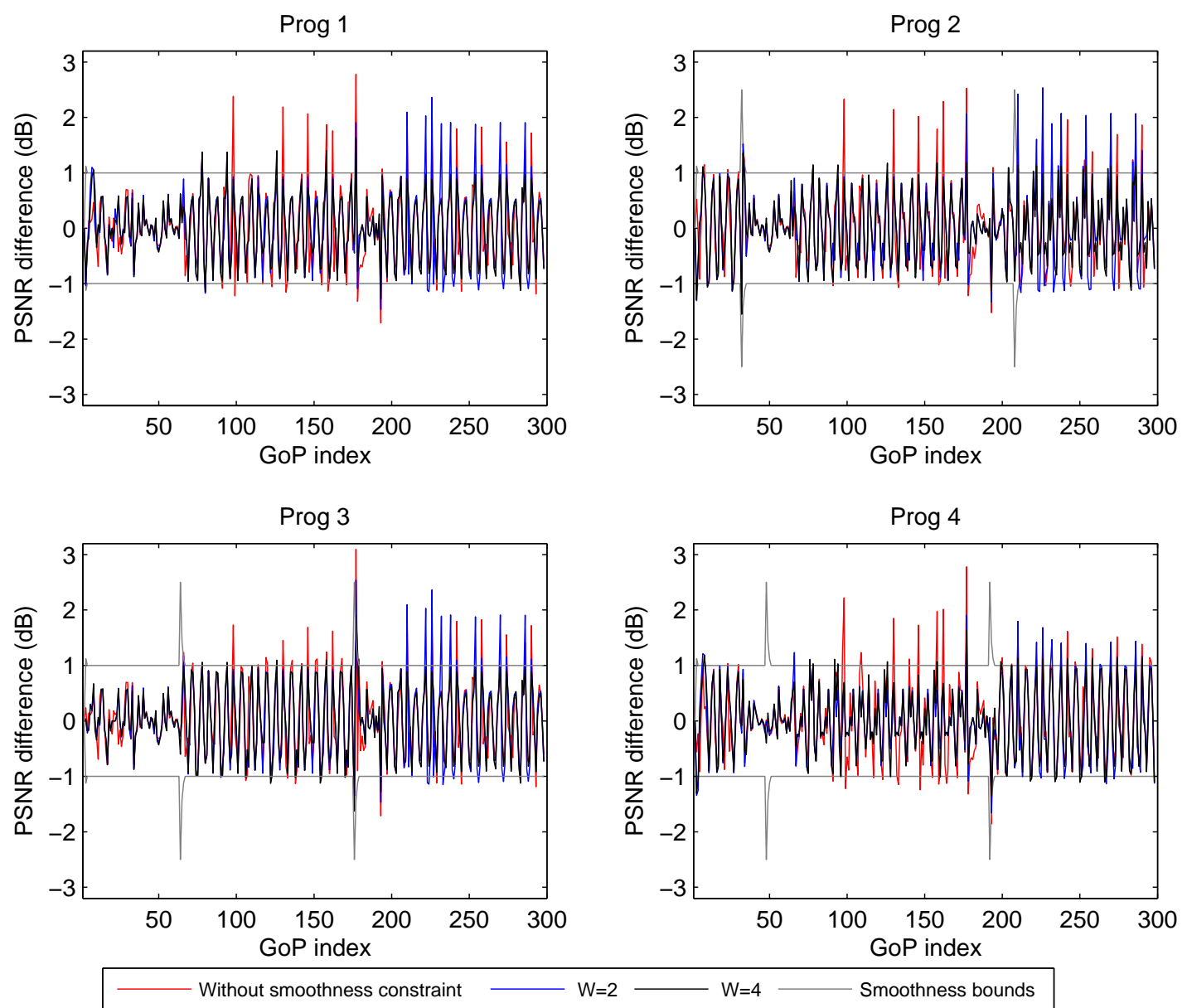

Figure 6. PSNR differences and bounds for the smoothness constraint, when $W=2$ and $W=4$

the overshoot. Figure 9(b) shows that $K_{\mathrm{i}}=0.01$ provides the best results. Finally $K_{\mathrm{d}}$ should provide some additional stability to the system. In Figure 9(c), $K_{\mathrm{d}}=0.2$ is a reasonable choice. Figure 9 shows some oscillating behavior after the transient phase. This is due (i) to the discrepancy between the model and the actual RD characteristics and (ii) to the fact that the QPs provided by the optimization process have to be rounded before being used by the video encoders. This type of oscillatory behavior due to quantized inputs has been considered in [62] and [63]. Using scalable video coders, such as H.264/SVC, combined with layer filtering instead of transcoding or encoding at various QPs would probably enhance the oscillating behavior. Scalable video coders based on allowing layer filtering do not provide a very fine rate adaptation, leading to an effect similar to a coarse quantization of the QPs, depending on the number of scalability layers.

2) Variable channel rate: This section considers a variable rate of the broadcast channel. The rate variations are modeled as a three-state Markov chain, each state representing a rate belonging to $\mathcal{R}^{c}=\{800,1000,1200\}$ kbits/s. The channel state transition probabilities are given in the following transition matrix

$$
P=\left[\begin{array}{ccc}
0.95 & 0.05 & 0 \\
0.025 & 0.95 & 0.025 \\
0 & 0.05 & 0.95
\end{array}\right]
$$

Notice that even when performing a predictive control, only the channel rate at time $j$ is assumed to be known and expected rates at future time instants are evaluated, see (7).

a) Rate and quality control: Figure 10 shows the encoding rates $R_{i j}^{\mathrm{e}}$ for each program, the total encoding rate $R_{j}^{\mathrm{e}}=\sum_{i=1}^{4} R_{i j}^{\mathrm{e}}$, and the total transmission rate $R_{j}^{\mathrm{t}}=\sum_{i=1}^{4} R_{i j}^{\mathrm{t}}$ for $W=2$ (a) and $W=4$ (b). In both cases $W=2$ and $W=4$, the encoding rate is efficiently updated to allow an full use of the available channel rate. The same values for the PID parameters as in Section VI-A1b have been used.

The proposed control system is robust to variations of the characteristics of the video contents and of the channel rate. When the channel rate varies, the system is able to update the encoding parameters so that the 

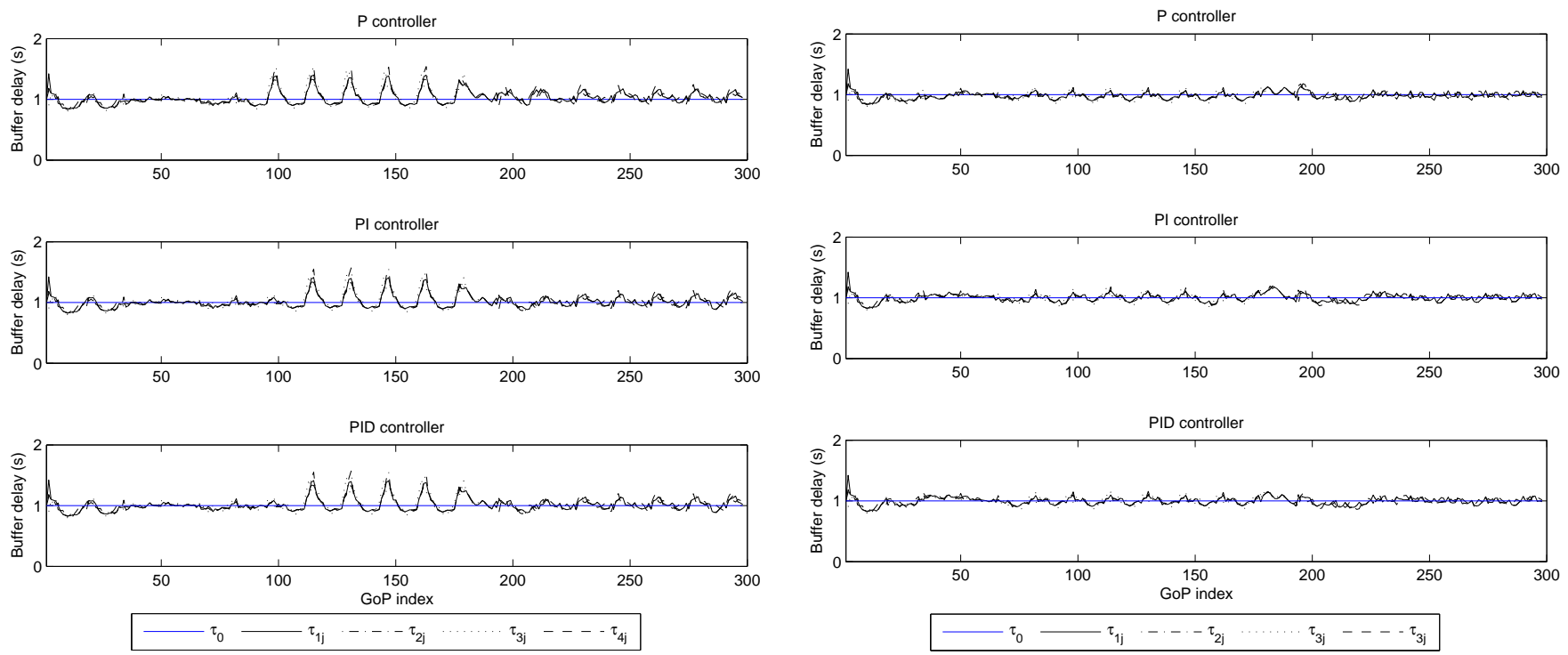

(a)

(b)

Figure 8. Buffering delay evolution of the 4 multiplexed programs using P, PI, and PID controller with $W=2$ (a) and $W=4$ (b) in the encoder control process with constant channel rate

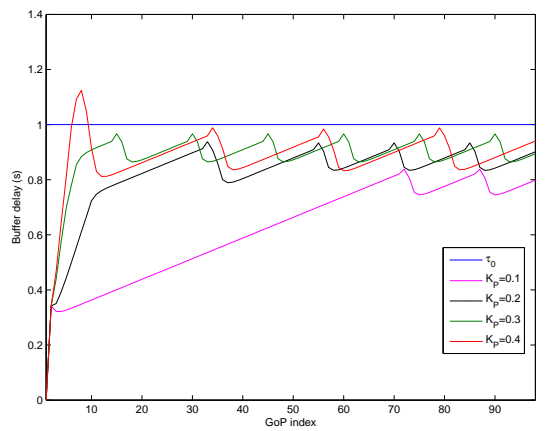

(a)

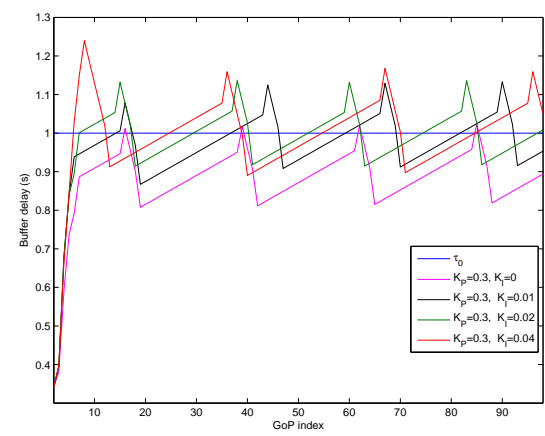

(b)

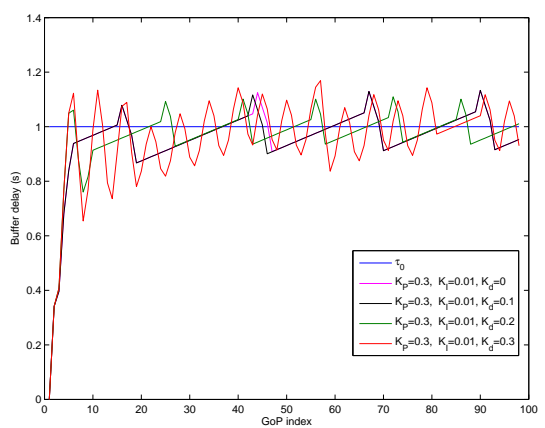

(c)

Figure 9. Tuning of the parameters of the PID controller: (a) P controller, (b) PI controller, (c) PID controller

total encoding rate satisfies the updated rate constraint and the smoothness and fairness constraints are satisfied. As in the constant channel rate case, $W=4$ provides a much lower jitter of the encoding rate than $W=2$.

The PSNR variations resulting from the encoder control process are represented in Figure 11 for the four multiplexed programs for $W=2$ (a) and $W=4$ (b). As in the constant channel rate case, the predictive control $(W=4)$ reduces the PSNR standard deviation from $2.15 \mathrm{~dB}$ without predictive control to $1.8 \mathrm{~dB}$. As already mentioned, the jitter in rate has a direct impact on the jitter in PSNR.

The performance in terms of $\Delta \tau, \sigma_{\tau}^{2}$, and $e$ for the buffer control are provided in Table IV, showing a good robustness of the PID parameters with respect to variations of the channel rate. The buffering delays in each buffer are represented in Figure 12 for $W=2$ (a) and $W=4$ (b) using P, PI, and PID controllers. Channel variations lead in this case to less differences between the P, PI, and PID controllers when $W=2$ and $W=4$ than in the constant channel rate case. Nevertheless, Figure 12 shows that the delay remains closer to the reference delay when $W=4$ than with $W=2$.

\section{B. Scenario 2: Statistical multiplexing using eight video programs in 4CIF format}

This section considers a typical application context presented in Section V-B concerning mobile TV service delivery over evolved MBMS standard. Eight video programs, each of $100 \mathrm{~s}$ long, extracted from real TV programs in 4CIF format are multiplexed. Video sequences correspond to a weather forecast, a soccer game, a video clip, a cartoon, a TV show, an interview, an extract of the trailer of Harry Potter. The videos are already encoded using MPEG-4 have been converted to 


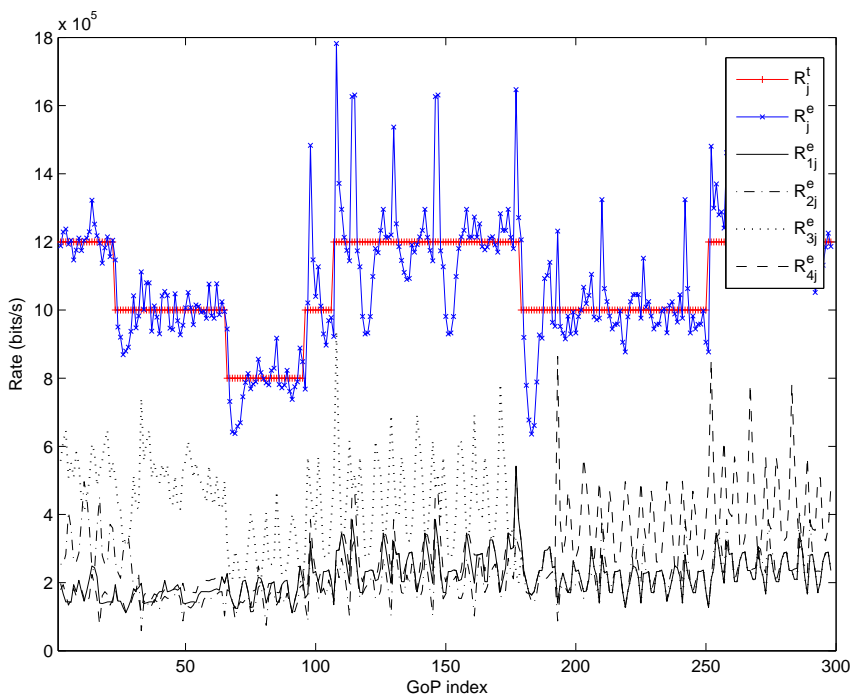

(a)

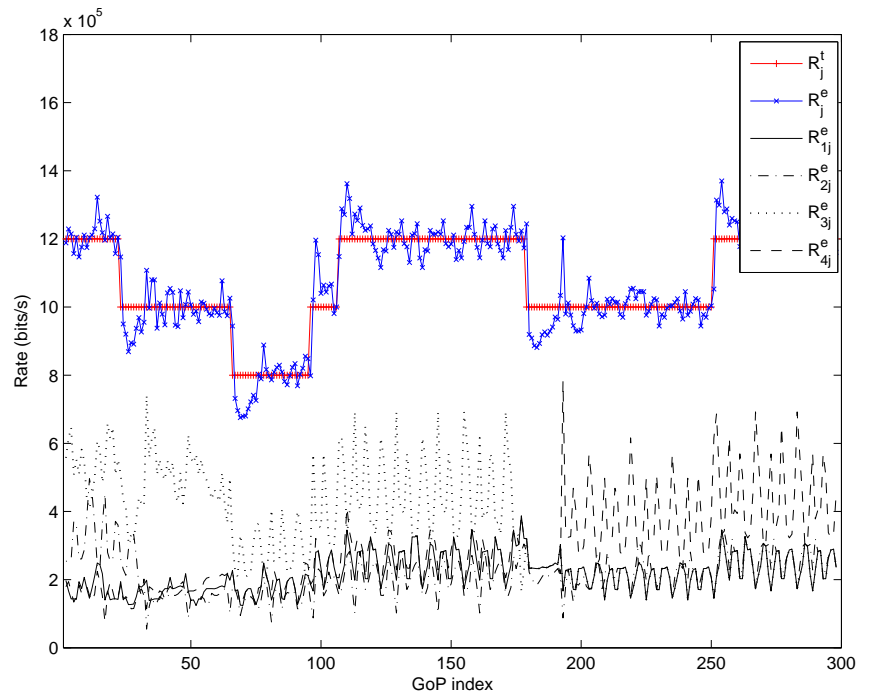

(b)

Figure 10. Evolution of the total transmission rates as well as individual and total encoding rates for the four multiplexed programs when $W=2$ (a) and $W=4$ (b) with variable channel rate

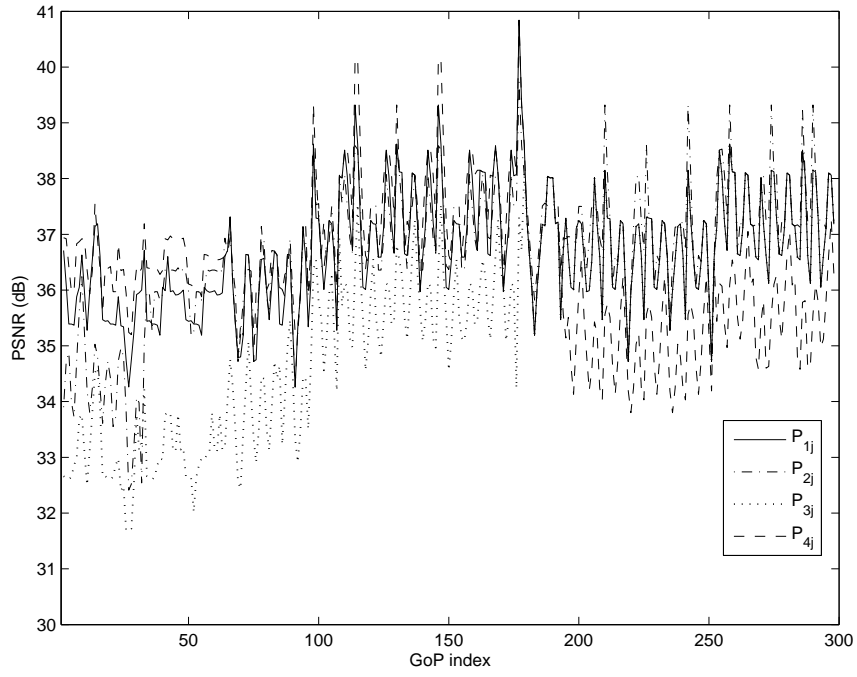

(a)

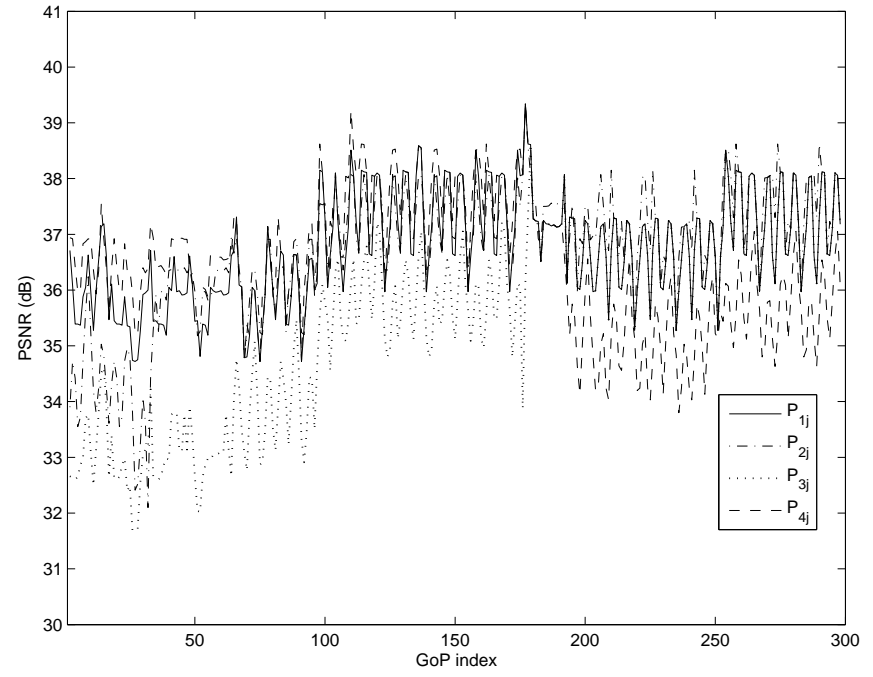

(b)

Figure 11. Evolution of the PSNR of the four multiplexed programs when $W=2$ (a) and $W=4$ (b) with variable channel rate

\begin{tabular}{|c|c|c|c|c|c|c|c|}
\hline$K_{\mathrm{p}}, K_{\mathrm{i}}, K_{\mathrm{d}}$ & \multicolumn{3}{|c|}{$W=2$} & $K_{\mathrm{p}}, K_{\mathrm{i}}, K_{\mathrm{d}}$ & \multicolumn{3}{c|}{$W=4$} \\
\hline & $\Delta \tau$ & $\sigma_{\tau}^{2}$ & $e$ & & $\Delta \tau$ & $\sigma_{\tau}^{2}$ & $e$ \\
\hline $0.2,0,0$ & 0.03 & 0.08 & 0.08 & $0.2,0,0$ & 0.01 & 0.03 & 0.03 \\
\hline $0.2,0.01,0$ & 0.001 & 0.13 & 0.13 & $0.2,0.01,0$ & 0.001 & 0.04 & 0.04 \\
\hline $0.2,0.01,0.01$ & 0.001 & 0.06 & 0.06 & $0.2,0.01,0.05$ & 0.001 & 0.03 & 0.03 \\
\hline
\end{tabular}

Table IV

SYSTEM PERFORMANCE IN TERMS OF $\Delta \tau$ AND $\sigma_{\tau}^{2}$ WHEN USING P, PI, AND PID CONTROLLERS FOR $W=2$ AND $W=4$ USING VARIABLE CHANNEL RATE 

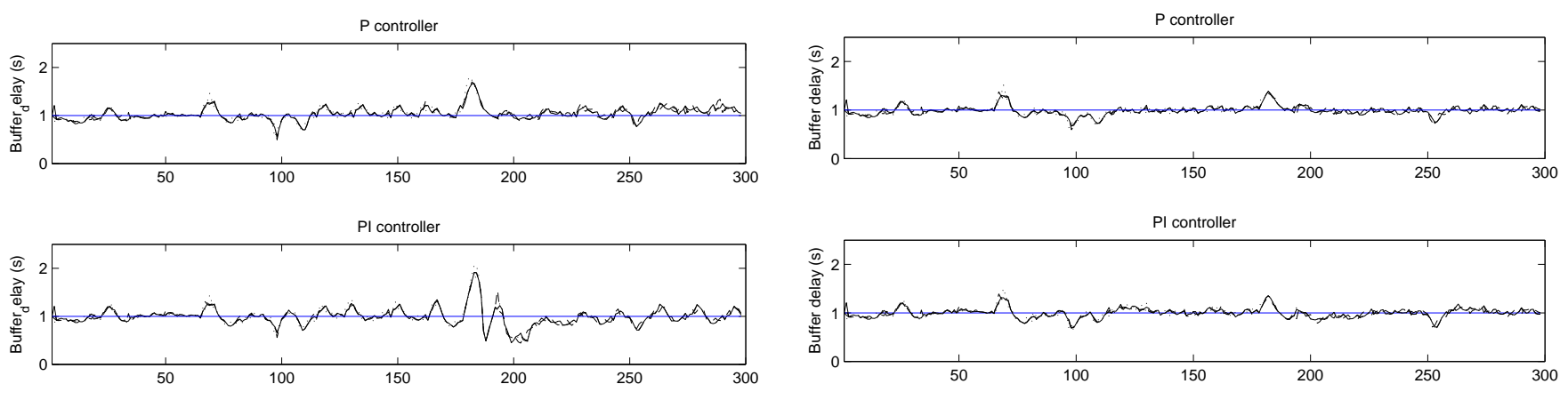

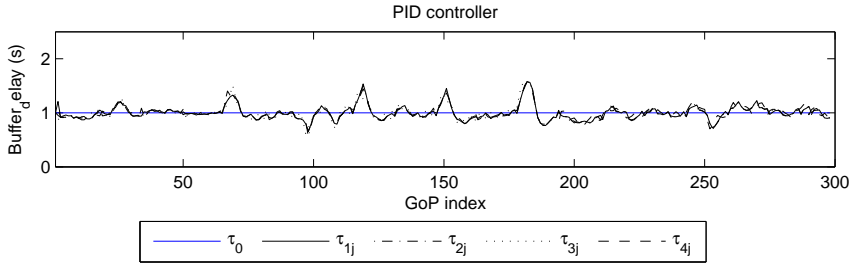

(a)

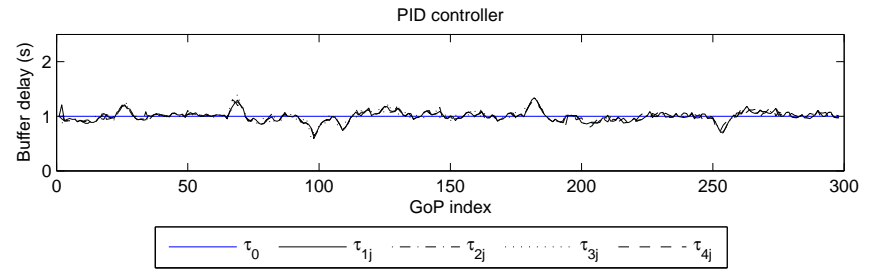

(b)

Figure 12. Buffering delay evolution of the four multiplexed programs using P, PI, and PID controller with $W=2$ (a) and $W=4(\mathrm{~b})$ in the encoder control process with variable channel rate

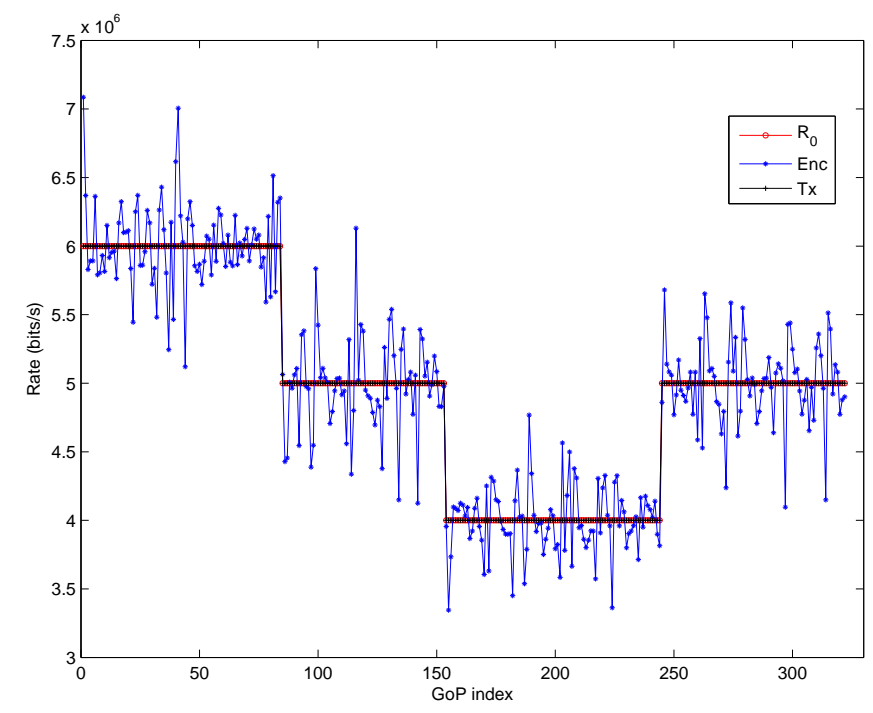

(a)

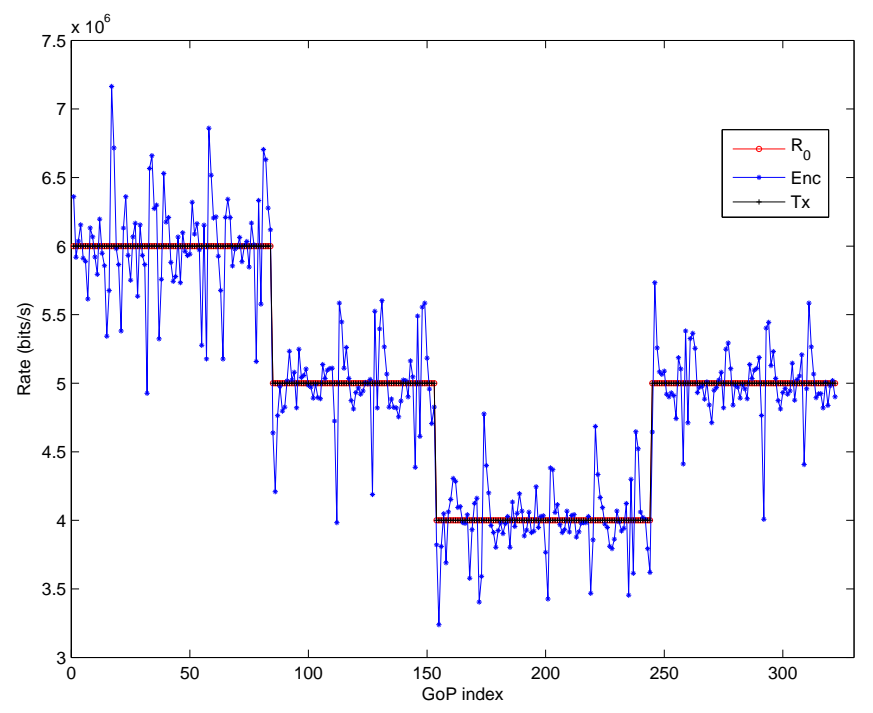

(b)

Figure 13. Total transmission rate, individual and total encoding rates for the eight multiplexed programs when $W=1$ (a) and $W=2$ (b)

YUV format using ffmpeg [64]. The eight videos are then processed with the proposed SM system.

For each program, video sequences are concatenated in loop at different time index. These time index correspond to abrupt scene changes and are assumed known in advance for each program. In addition, the considered video sequences contain a lot of variation over the time. These variations are not detected in our simulation. Thus, in order to better satisfy the smoothness constraint, the encoder rate constraint is set with $\varepsilon=0.1$ allowing larger variation in the encoder rate and less variation in the PSNR.

The minimum tolerated PSNR is $P_{\min }=30 \mathrm{~dB}$. The PSNR variation and discrepancy bounds are $\Delta P_{\max }^{s}=$ $2.5 \mathrm{~dB}, \Delta P_{\min }^{s}=1 \mathrm{~dB}, \Delta P_{\max }^{\mathrm{f}}=3 \mathrm{~dB}$, and $\Delta P_{\min }^{\mathrm{f}}=$ $2 \mathrm{~dB}$. The damping ratio is $\lambda=1.25$, leading to a negligible relaxation of the PSNR bounds after 1 to 2 VUs. The maximum buffer size in bits is $B_{\max }=2$ Mbits.

The reference delay is taken as $\tau_{0}=0.5 \mathrm{~s}$ since MBMS service shall add no more than 1 second delay when switching between different TV streams [54]. The rate variations are modeled as a three-state Markov 


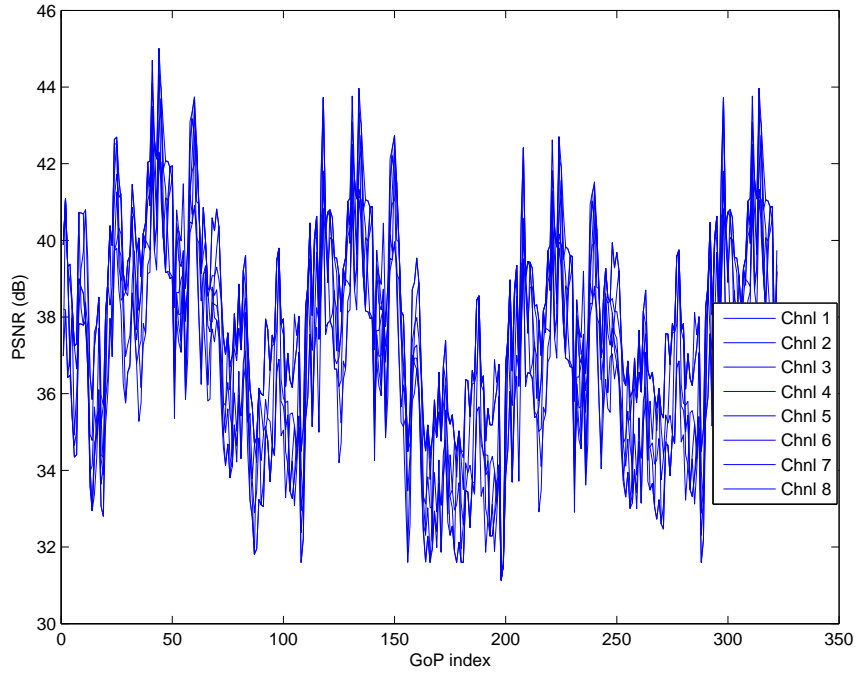

(a)

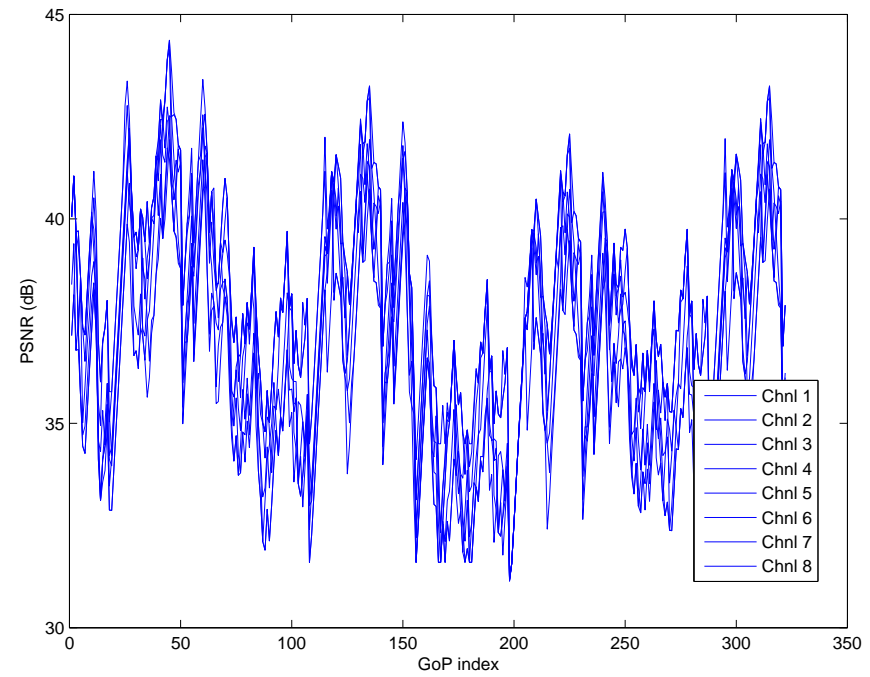

(b)

Figure 14. PSNR of the eight multiplexed programs when $W=1$ (a) and $W=2$ (b)

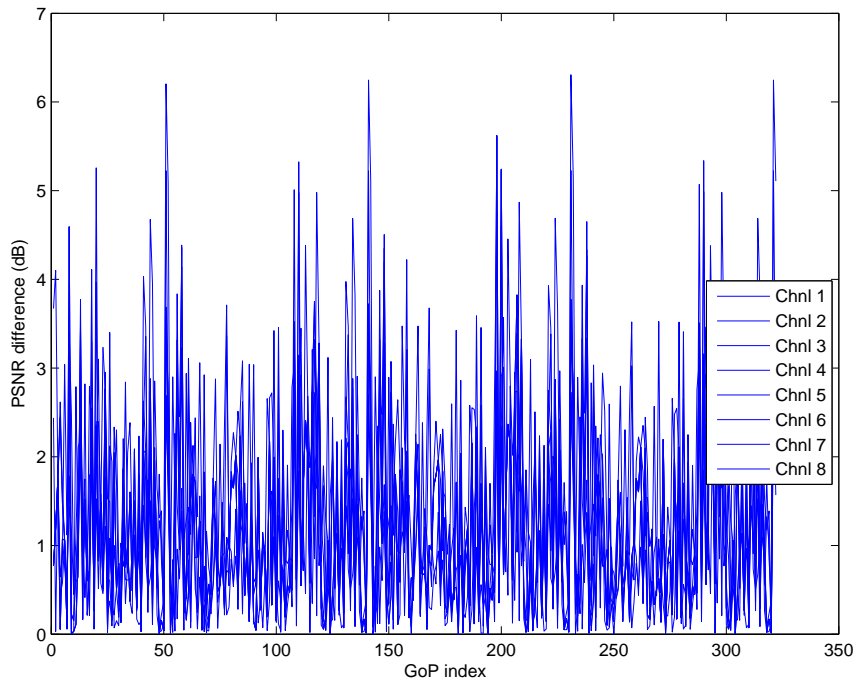

(a)

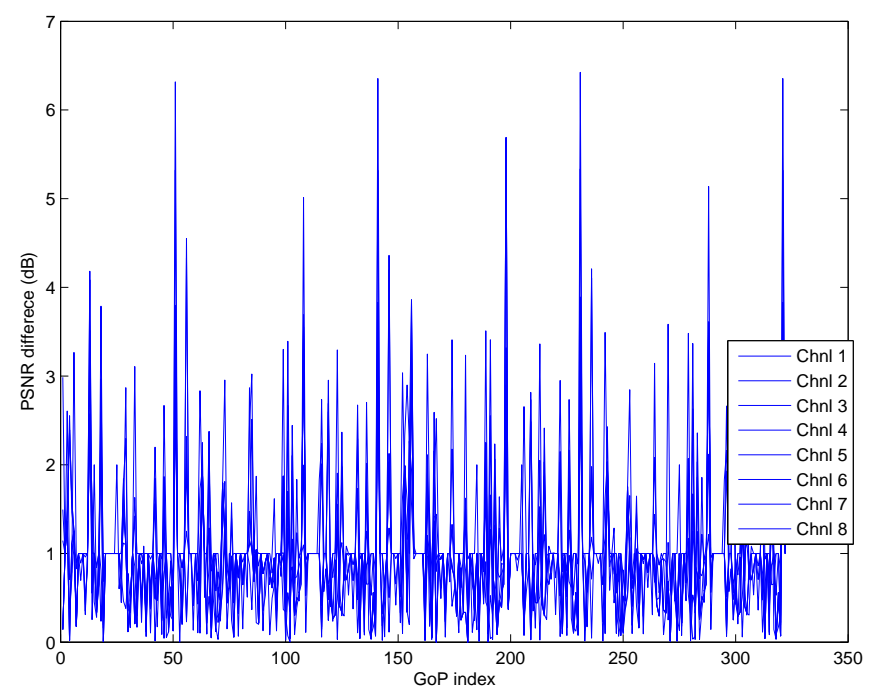

(b)

Figure 15. PSNR differences of the eight multiplexed programs when $W=1$ (a) and $W=2$ (b)

chain, each state representing a rate belonging to $\mathcal{R}^{c}=$ $\{4,5,6\} \mathrm{Mbits} / \mathrm{s}$. These rates are of the same order as those considered in [15]. The channel state transition probabilities are those of (27).

We compare the case when the video quality smoothness constraint is addressed $W>1$ and when the smoothness constraint is not taken into account (this case is again denoted as $W=1$ ). We observed that in these simulations, taking $W>2$ with the same values of $\Delta P_{i j}^{\mathrm{f}}$ and $\Delta P_{i j}^{\mathrm{s}}$ does not provide additional benefit in terms of variations of the PSNR. This is due to the high and rapid activity variation in the considered video sequences. In this section we compare the case where the smoothness constraint is not addressed $W=1$ and the case when the video quality smoothness constraint is addressed using previously encoded GoPs $W=2$.

Again, only the channel rate at time $j$ is assumed to be known. The parameters of the PID controller for the feedback from the buffer controller to the encoder controller are set to $\left(K_{\mathrm{p}}, K_{\mathrm{i}}, K_{\mathrm{d}}\right)=(0.35,0.2,0.3)$ leading to a minimum difference between the measured delay and the targeted delay and small variations as shown in Table V.

Figure 13 shows the encoding rates $R_{i j}^{\mathrm{e}}$ for each program, the total encoding rate $R_{j}^{\mathrm{e}}=\sum_{i=1}^{8} R_{i j}^{\mathrm{e}}$, and the total transmission rate $R_{j}^{\mathrm{t}}=\sum_{i=1}^{8} R_{i j}^{\mathrm{t}}$ for $W=1$ (a) and $W=2$ (b) using a PID controller. In both cases $W=1$ and $W=2$, the encoding rate is efficiently 


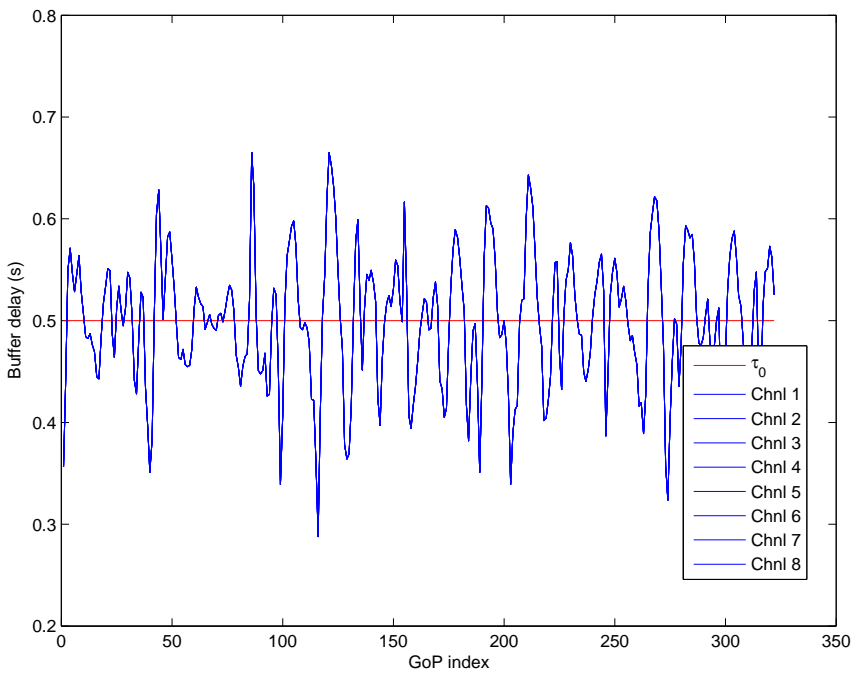

(a)

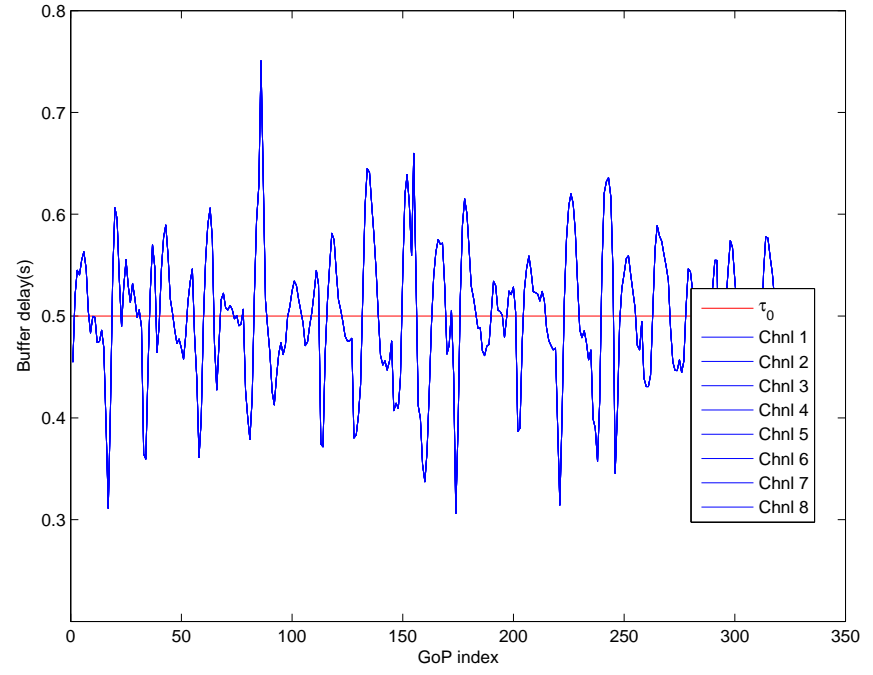

(b)

Figure 16. Buffering delay evolution of the eight multiplexed programs with $W=1$ (a) and $W=2$ (b)

\begin{tabular}{|c|c|c|c|c|}
\hline$K_{\mathrm{p}}, K_{\mathrm{i}}, K_{\mathrm{d}}$ & \multicolumn{2}{|c|}{$W=1$} & \multicolumn{2}{c|}{$W=2$} \\
\hline & $\Delta \tau$ & $\sigma_{\tau}^{2}$ & $\Delta \tau$ & $\sigma_{\tau}^{2}$ \\
\hline $0.35,0,0$ & 0.260 & 0.005 & 0.226 & 0.006 \\
\hline $0.35,0.2,0$ & 0.030 & 0.003 & 0.062 & 0.007 \\
\hline $0.35,0.2,0.3$ & 0.025 & 0.002 & 0.048 & 0.005 \\
\hline
\end{tabular}

Table V

SYSTEM PERFORMANCE IN TERMS OF $\Delta \tau$ AND $\sigma_{\tau}^{2}$ WHEN USING P, PI, AND PID CONTROLLERS FOR $W=1$ AND $W=2$ USING EIGHT VIDEO PROGRAMS

updated to allow an full use of the available channel rate.

The PSNR variations resulting from the encoder control process are represented in Figure 14 for the eight multiplexed programs for $W=1$ (a) and $W=2$ (b) using PID controller. In Figure 15, the PSNR difference between successive GoPs is represented. Due to the high activity level of the considered video sequences, the $1 \mathrm{~dB}$ smoothness constraint is satisfied $68 \%$ of the time. As in the previous scenario, using the smoothness constraint reduces the PSNR standard deviation from $6.38 \mathrm{~dB}$ when the smoothness constraint is not considered to $5.21 \mathrm{~dB}$.

The buffering delays in each buffer are represented in Figure 16 for $W=1$ (a) and $W=2$ (b). Relaxing the encoder rate constraint, leads to more fluctuations of the encoding rate, which results in fluctuations of buffering delay. The use of the integral term allows reducing the discrepancy between the delay in the buffers and the reference delay, see Table V.

\section{CONCLUSIONS}

A predictive controller for a SM system using H.264/AVC video encoders has been presented in the context of video broadcasting. The proposed system performs a closed-loop regulation of the encoders and the buffers using a PID feedback. Control accounts for the channel rate variations by distributing the available channel rate among the encoders while satisfying minimum quality, smoothness, and fairness constraints. A similar and small buffering delay for all multiplexed programs is also targeted.

The performance of the proposed system has been evaluated via simulations at GoP level and compared with a reference control scheme where only regulation with respect to the past GoP is performed. Experimental results with constant and variable channel rate show that thanks to the predictive and to the closed-loop control of the encoders and of the buffers, the channel is efficiently used, the video quality constraints are satisfied as well as the constraints on the buffering delays. Moreover, predictive control decreases the intra-program quality variations compared to the non-predictive control.

The adaptation of the proposed SM at the frame level, as in [17], will be addressed in future work. A closed-loop rate and buffer control at the frame level requires dependent $\mathrm{RD}$ models, such as those described in [24], [65], or [44]. Such models $R_{i j}\left(Q_{i j}, Q_{i j-1}\right)$ and $D_{i j}\left(Q_{i j}, Q_{i j-1}\right)$ take into account the impact of 
the chosen QP in the reference frame on the rate and the distortion of its corresponding predicted frame. The price to be paid is a much increased modeling complexity than with a GoP-level control.

Extensions to scalable video coders may also be considered. This would facilitate the obtention of the RD model for each video sequence. Using layer filtering, an easy rate and quality adaptation is possible. The price to be paid is a quite large granularity of the control input, which may lead to increased oscillation behavior of the buffering delays, as already noticed when the QPs are rounded before being provided to the encoders or transcoders.

\section{REFERENCES}

[1] ETSI, "Digital video broadcasting (DVB); DVB-SH implementation guidelines," ETSI TS, Standard, Dec 2008.

[2] ETSI, "Multimedia Broadcast/Multicast service. (MBMS); UTRAN/GERAN requirements," 3GPP TR 25.992-140, Tech. Rep., jun. 2005.

[3] R. Koenen, "MPEG-4 overview," ISO/IEC JTC1/SC29/WG11, Tech. Rep., March 2002.

[4] T. Wiegand, G. Sullivan, G. Bjontegaard, and A. Luthra, "Overview of the H.264/AVC video coding standard," IEEE Trans. on Circuits and Systems for Video Technology, vol. 13, no. 7, pp. 560-576, July 2003.

[5] H. Schwarz, D. Marpe, and T. Wiegand, "Overview of the scalable video coding extension of the H.264/AVC standard," IEEE Trans. on Circuits and Systems for Video Technology In Circuits and Systems for Video Technology,, vol. 17, no. 9, pp. 1103-1120, September 2007.

[6] Q. Chengsheng, C. Guobin, and L. Jilin, "An efficient twopass VBR encoding algorithm for H.264," in Int. Conference on Communications Circuits and Systems Proc., vol. 1, June 2006, pp. 118-122.

[7] K. Chandra, The Wiley Encyclopedia of Telecommunications. Wiley, 2002, ch. Statistical Multiplexing.

[8] G. Valenzise, M. Tagliasacchi, and S. Tubaro, "A smoothed, minimum distortion-variance rate control algorithm for multiplexed transcoded video sequences," in Proc. Int. Workshop on Mobile Video. New York, NY, USA: ACM, 2007, pp. 55-60.

[9] M. Jacobs, J. Barbarien, S. Tondeur, R. Van de Walle, T. Paridaens, and P. Schelkens, "Statistical multiplexing using SVC," in IEEE International Symposium on Broadband Multimedia Systems and Broadcasting, march 2008, pp. 1-6.

[10] M. Tagliasacchi, G. Valenzise, and S. Tubaro, "Minimum variance optimal rate allocation for multiplexed H.264/AVC bitstreams," IEEE Trans. on Image Processing, vol. 17, no. 7, pp. 1129-1143, July 2008.

[11] M. Rezaei, I. Bouazizi, and M. Gabbouj, "Implementing statistical multiplexing in DVB-H," International Journal of Digital Multimedia Broadcasting, p. 15, April 2009, article ID 261231.

[12] X. Wang and Y. Zhang, "Optimal video stream multiplexing in MBSFN," in IEEE International Conference on Communications Technology and Applications, oct. 2009, pp. $360-365$.

[13] A. Elwalid and D. Mitra, "Statistical multiplexing with loss priorities in rate-based congestion control of high-speed networks," IEEE Trans. on Communications, vol. 42, no. 11, pp. 2989 -3002, nov 1994.

[14] L. Wang and A. Vincent, "Joint rate control for multi-program video coding," IEEE Trans. on Consumer Electronics, vol. 42, no. 3, pp. 300-305, Aug 1996.
[15] V. Vukadinovic and J. Huschke, "Statistical multiplexing gains of H.264/AVC video in E-MBMS," in International Symposium on Wireless Pervasive Computing, May 2008, pp. $468-474$.

[16] M. Rezaei, I. Bouazizi, and M. Gabbouj, "Joint video coding and statistical multiplexing for broadcasting over DVB-H channels," IEEE Transactions on Multimedia, vol. 10, no. 8, pp. $1455-1464$, dec. 2008.

[17] Z. He and D. O. Wu, "Linear rate control and optimum statistical multiplexing for H.264 video broadcast," IEEE Trans. on Multimedia, vol. 10, no. 7, pp. 1237-1249, November 2008.

[18] M. Perkins and D. Arnstein, "Statistical multiplexing of multiple MPEG-2 video programs in a single channel," SMPTE journal, vol. 104, no. 9, pp. 596-599, 1995.

[19] Z. Zhang, S. Nelakuditi, R. Aggarwal, and R. Tsang, "Efficient selective frame discard algorithms for stored video delivery across resource constrained networks," Real-Time Imaging, vol. 7, no. 3, pp. 255-273, June 2001.

[20] T.-L. Lin, Y. Zhi, S. Kanumuri, P. Cosman, and A. Reibman, "Perceptual quality based packet dropping for generalized video gop structures," in IEEE International Conference on Acoustics, Speech and Signal Processing, ser. ICASSP '09, 2009, pp. 781784.

[21] S. Ma, W. Gao, and Y. Lu, "Rate-distortion analysis for H.264/AVC video coding and its application to rate control," IEEE Trans. on Circuits and Systems for Video Technology, vol. 15, no. 12, pp. 1533-1544, December 2005.

[22] S. K. Srinivasan, J. Vahabzadeh-Hagh, and M. Reisslein, "The effects of priority levels and buffering on the statistical multiplexing of single-layer H.264/AVC and SVC encoded video streams," IEEE Trans. on Broadcasting, vol. 56, no. 3, pp. 281 $-287,2010$.

[23] E. Maani and A. Katsaggelos, "Unequal error protection for robust streaming of scalable video over packet lossy networks," IEEE Trans. on Circuits and Systems for Video Technology, vol. 20, no. 3, pp. $407-416$, march 2010.

[24] J. Lin and A. Ortega, "Bit-rate control using piecewise approximated rate-distortion characteristics," IEEE Trans. on Circuits Syst. Video Technol, vol. 8, pp. 446-459, 1998.

[25] L. Luo, J. Xu, S. Li, and Z. Zhuang, "Rate control with smoothed temporal distortion for a 3D embedded wavelet video coder," in Proc. IEEE Int. Conf. Info., Commu. \& Sig. Process, Singapore, Oct. 2001.

[26] A. Munteanu, Y. Andreopoulos, M. van der Schaar, P. Schelkens, and J. Cornelis, "Control of the distortion variation in video coding systems based on motion compensated temporal filtering," in International Conference on Image Processing, vol. 2, sept. 2003, pp. II - 61-4 vol.3.

[27] X. Kang, J. Lan, L. Liu, and X. Zhuang, "SNR-based bit allocation in video quality smoothing," in Advances in Multimedia Information Processing - PCM 2006, ser. Lecture Notes in Computer Science, 2006, vol. 4261, pp. 989-998.

[28] L. Böröczky, A. Y. Ngai, and E. F. Westermann, "Statistical multiplexing using MPEG-2 video encoders," IBM Journal of Research and Development, vol. 43, no. 4, pp. 511-520, 1999.

[29] J. Zdepski, D. Raychaudhuri, and K. Joseph, "Statistically based buffer control policies for constant rate transmission of compressed digital video," IEEE Trans. on Communications, vol. 39, no. 6, pp. 947 -957, jun 1991.

[30] M. Mandjes, D. Mitra, and W. Scheinhardt, "Models of network access using feedback fluid queues," Queueing Syst. Theory Appl., vol. 44, no. 4, pp. 365-398, 2003.

[31] L. Wing-Cheong and L. San-Qi, "Statistical multiplexing and buffer sharing in multimedia high-speed networks: a frequencydomain perspective," IEEE/ACM Trans. on Networking, vol. 5, no. 3, pp. $382-396$, jun 1997. 
[32] N. Changuel, B. Sayadi, and M. Kieffer, "Joint encoder and buffer control for statistical multiplexing of multimedia contents," in IEEE Global Communications Conference: Globecom, 2010, pp. $1-6$.

[33] ITU-T, "Objective perceptual multimedia video quality measurement in the presence of a full reference," ITU-T Rec. J.247 (08/08), Tech. Rep. Rec. J.247, 2008.

[34] G. J. Sullivan and T. Wiegand, "Rate-distortion optimization for video compression," IEEE Signal Processing Magazine, vol. 15, no. 6, pp. 74-90, November 1998.

[35] G. Winkler, Image Analysis, Random Fields and Dynamic Monte Carlo Methods: A Mathematical Introduction, Second edition, 2nd ed., S. Verlag, Ed. Springer, 2005, vol. 27 of Applications of Mathematics.

[36] W. Ding and B. Liu, "Rate control of MPEG video coding and recording by rate-quantization modeling," IEEE trans. on Circuits and Systems for Video Technology, vol. 6, no. 1, pp. $12-20$, february 1996.

[37] H. Hang and J. Chen, "Source model for transform video coder and its application I: Fundamental theory," IEEE Trans. on Circuits and Systems for Video Technology, vol. 7, no. 2, pp. 287-298, April 1997.

[38] N. Kamaci, Y. Altunbasak, and R. Mersereau, "Frame bit allocation for the H.264/AVC video coder via Cauchy-densitybased rate and distortion models," IEEE Trans on Circuits and Systems for Video Technology, vol. 15, no. 8, pp. 994-1006, Aug. 2005.

[39] H. Shen, X. Sun, F. Wu, and S. Li, "Rate-distortion optimization for fast hierarchical B-picture transcoding," Proc. IEEE International Symposium on Circuits and Systems, pp. 5279-5282, 2006.

[40] X. Zhu, P. Agrawal, J. Pal Singh, T. Alpcan, and B. Girod, "Rate allocation for multi-user video streaming over heterogenous access networks," in ACM Multimedia, Germany, September 2007, pp. 37-46.

[41] Y. Liu, Z. Li, and Y. Soh, "A novel rate control scheme for low delay video communication of H.264/AVC standard," IEEE Trans. on Circuits and Systems for Video Technology, vol. 17, no. 1, pp. 68-78, January 2007.

[42] N. Changuel, B. Sayadi, and M. Kieffer, "Predictive control for efficient statistical multiplexing of digital video programs," in Int. Packet Video Workshop, May 2009, pp. 1-9.

[43] X. Minghui, A. Vetro, S. Huifang, and L. Bede, "Rate-distortion optimized bit allocation for error resilient video transcoding," in ISCAS, vol. 3, May 2004, pp. III-945-8 Vol.3.

[44] N. Changuel, B. Sayadi, and M. Kieffer, "H.264/AVC inter frame rate distortion dependency analysis based on independent regime switching AR models," in IEEE International Conference on Acoustics, Speech, and Signal Processing, Mars 2010.

[45] Z. He and S. K. Mitra, "A linear source model and a unified rate control algorithm for DCT video coding," IEEE Transactions on Circuits and Systems for Video Technology, vol. 12, no. 11, pp. 970-982, November 2002.

[46] L. Liu and X. Zhuang, "A novel square root rate control algorithm for H.264/AVC encoding," in IEEE International Conference on Multimedia and Expo, june 2009, pp. 814 -817.

[47] Z. Li, W. Gao, F. Pan, S. Ma, K. Pang Lim, G. Feng, X. Lin, S. Rahardja, H. Lu, and Y. Lu, "Adaptive basic unit layer rate control for JVT," JVT-H014, Geneva, Tech. Rep., 2003.

[48] H. Wang and S. Kwong, "A rate-distortion optimization algorithm for rate control in H.264," in IEEE International Conference on Acoustics, Speech and Signal Processing, vol. 1, 2007, pp. I-1149 -I-1152.

[49] J. Yang, Q. Dai, W. Xu, and R. Ding, "A rate control algorithm for MPEG-2 to H.264 real-time transcoding," Visual Communications and Image Processing, vol. 5960, pp. 1995-2003, 2005.
[50] C.-H. Chu, H.-P. Hung, and M.-S. Chen, "Variant bandwidth channel allocation in the data broadcasting environment," in International Conference on Mobile Data Management, may 2007, pp. $94-101$

[51] T. I. Sesia, S. and and M. Baker, LTE, The UMTS Long Term Evolution: From Theory to Practice. Wiley, Feb. 2009.

[52] M. Jolfaei, D. Kreuer, O. Maly, U. Quernheim, and W. Kremer, "Time variant models for satellite channel," in IEEE Vehicular Technology Conference, vol. 1, 1992, pp. 143 - 146.

[53] C. Martin, A. Geurtz, and B. Ottersten, "File based mobile satellite broadcast systems: Error rate computation and QoS based design," in Proceedings IEEE Vehicular Technology Conference, 2004, vol. 6, pp. 4017-4021.

[54] ARIB STD-T63-22.246 V8.5.0, "Multimidia Broadcast/Multicast Service (MBMS) user services stage 1 (release 8)," 3GPP TS, Tech. Rep., 2008.

[55] K. Ström and T. Hägglund, Advanced PID Control. USA: ISA, Research Triangle Park, 2005.

[56] G. M. Van Der Zalm, Examination board tuning of PID type controllers: Literature overview. Eindhoven : Technische Universiteit Eindhoven, 2008.

[57] K.-B. Kim and H.-J. Kim, "Back-pressure buffering scheme to improve the cell loss property on the output buffered atm switch," in IEEE Conference on Local Computer Networks, 1996, pp. $242-248$.

[58] H.-M. Sun, Y.-C. Lin, and L. Shu, "The impact of varying frame rates and bit rates on perceived quality of low/high motion sequences with smooth/complex texture," Multimedia Systems, vol. 14, no. 1, pp. 1-13, 2008.

[59] W. Fernando and K. Loo, "Abrupt and gradual scene transition detection in MPEG-4 compressed video sequences using texture and macroblock information," in International Conference on Image Processing, vol. 3, 2004, pp. 1589-1592.

[60] J.-W. Kim, S-M. Byun and C.-S. Won, "A scene change detection in H.264/AVC compression domain," in Advances in Multimedia Information Processing, vol. 3768, 2005, pp. 10721082.

[61] S. Moiron, R. Razavi, M. Fleury, and M. Ghanbari, "Statistical multiplexing of transcoded IPTV streams based on content complexity," in Int. Conf. on Multimedia Communications (MOBIMEDIA2010), 2010.

[62] A. Picasso, B.; Bicchi, "On the stabilization of linear systems under assigned I/O quantization,", IEEE Transactions on Automatic Control, vol. 52, no. 10, pp. 1994-2000, 2007.

[63] A. H. C. Gosline, V. Hayward, and H. Michalska, "Ineluctability of oscillations in systems with digital implementation of derivative feedback," Automatica, p. in press, 2011.

[64] [Online]. Available: http://ffmpeg.org/ffmpeg.html

[65] X. Minghui, A. Vetro, S. Huifang, and L. Bede, "Rate distortion optimized bit allocation for error resilient video transcoding," in IEEE International Symposium on Circuits and Systems, vol. 5, 2004, pp. 945-948. 


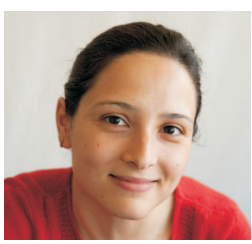

Nesrine Changuel (IEEE S'09 - M'12) received her B.S. degree in 2006 and Eng. degree and M.S. degree in electrical engineering in 2008 from the Ecole Nationale Supérieure d'Electronique et de Radioélectricitée de Grenoble, France. She obtained a PhD degree in Control and Signal Processing in 2011 from the Paris-Sud University, Orsay. She is currently working as a research engineer in Alcatel Lucent Bell Labs. Her current research interests are in the areas of video coding, Statistical multiplexing of video programs, scalable video, Rate and Distortion model, resource allocation and scheduling, Markov decision processes (MDPs), and reinforcement learning. She is a Member of the IEEE.

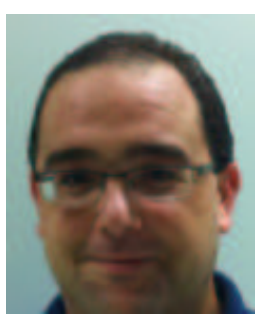

Bessem Sayadi is a member of technical staff at Alcatel-Lucent Bell Labs, France. $\mathrm{He}$ is member of Alcatel-Lucent Technical Academy, since 2008. He received M.Sc. (00) and Ph.D. (03) degrees in Automatic and Signal processing from Supélec, ParisSud University, with highest distinction. $\mathrm{He}$ worked previously as a postdoctoral fellow in the National Centre for Scientific Research (CNRS), and as a senior researcher engineer in Orange Labs. His main research interests are in the area of broadcast technology (DVB, 3GPP), video coding and transport, and content oriented networks. He has authored over 30 publications in journal and conference proceedings and serves as a regular reviewer for several technical journals and conferences. He holds 6 patents and has ten patent applications pending in the area of video coding and wireless communications. He serves or has served as a lecturer in Supélec, ENSEA, and Dauphine University.

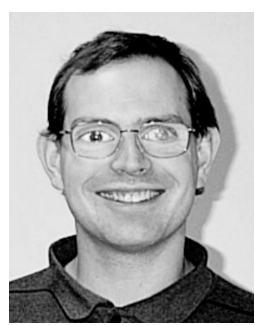

Michel Kieffer (IEEE M'02 - SM'07) received in 1995 the Agrégation in Applied Physics at the Ecole Normale Supérieure de Cachan. He obtained a PhD degree in Control and Signal Processing in 1999, and the Habilitation à Diriger des Recherches degree in 2005, both from the Paris-Sud University, Orsay.

Michel Kieffer is an associate professor in signal processing for communications at the Paris-Sud University and a researcher at the Laboratoire des Signaux et Systèmes, Gif-sur-Yvette. Since 2009, he is also invited professor at the Laboratoire Traitement et Communication de l'Information, Télécom ParisTech, Paris.

His research interests are in signal processing for multimedia, communications, and networking, distributed source coding, network coding, joint source-channel coding and decoding techniques, joint source-network coding. Applications are mainly in the reliable delivery of multimedia contents over wireless channels. He is also interested in guaranteed and robust parameter and state bounding for systems described by non-linear models in a bounded-error context.

Michel Kieffer is co-author of more than 120 contributions in journals, conference proceedings, or books. He is one of the coauthor of the book Applied Interval Analysis published by SpringerVerlag in 2001 and of the book Joint source-channel decoding: A crosslayer perspective with applications in video broadcasting published by Academic Press in 2009. He is associate editor of Signal Processing since 2008, of the Journal of Communication and Information Systems since 2011 and of the IEEE Transactions on Communications since 2012. In 2011, Michel Kieffer became junior member of the Institut Universitaire de France. 